\title{
Pointwise Bounds and Blow-up for Nonlinear Fractional Parabolic Inequalities
}

\author{
Steven D. Taliaferro \\ Department of Mathematics \\ Texas A\&M University \\ College Station, TX 77843-3368 \\ USA \\ stalia@math.tamu.edu
}

\begin{abstract}
We investigate pointwise upper bounds for nonnegative solutions $u(x, t)$ of the nonlinear initial value problem

$$
\begin{gathered}
0 \leq\left(\partial_{t}-\Delta\right)^{\alpha} u \leq u^{\lambda} \quad \text { in } \mathbb{R}^{n} \times \mathbb{R}, n \geq 1, \\
u=0 \quad \text { in } \mathbb{R}^{n} \times(-\infty, 0)
\end{gathered}
$$

where $\lambda$ and $\alpha$ are positive constants. To do this we first give a definition-tailored for our study of (0.1), (0.2) - of fractional powers of the heat operator $\left(\partial_{t}-\Delta\right)^{\alpha}: Y \rightarrow X$ where $X$ and $Y$ are linear spaces whose elements are real valued functions on $\mathbb{R}^{n} \times \mathbb{R}$ and $0<\alpha<\alpha_{0}$ for some $\alpha_{0}$ which depends on $n, X$ and $Y$.

We then obtain, when they exist, optimal pointwise upper bounds on $\mathbb{R}^{n} \times(0, \infty)$ for nonnegative solutions $u \in Y$ of the initial value problem (0.1), (0.2) with particular emphasis on those bounds as $t \rightarrow 0^{+}$and as $t \rightarrow \infty$.

2010 Mathematics Subject Classification. 35B09, 35B33, 35B44, 35B45, 35K58, 35R11, 35R 45.

Keywords. Blow-up, Pointwise bounds, Fractional heat operator, Parabolic.
\end{abstract}

\section{Introduction}

In this paper we study pointwise upper bounds for nonnegative solutions $u(x, t)$ of the nonlinear inequalities

$$
0 \leq\left(\partial_{t}-\Delta\right)^{\alpha} u \leq u^{\lambda} \quad \text { in } \mathbb{R}^{n} \times \mathbb{R}, n \geq 1,
$$

satisfying the initial condition

$$
u=0 \quad \text { in } \mathbb{R}^{n} \times(-\infty, 0)
$$

where $\lambda$ and $\alpha$ are positive constants.

To do this, we first give in Section 2 a definition-appropriate for our analysis of the initial value problem (1.1), (1.2) - of fractional powers of the heat operator

$$
\left(\partial_{t}-\Delta\right)^{\alpha}: Y \rightarrow X
$$

where $\Delta$ is the Laplacian with respect to $x \in \mathbb{R}^{n}, X$ and $Y$ are linear spaces whose elements are real valued functions on $\mathbb{R}^{n} \times \mathbb{R}$, and $0<\alpha<\alpha_{0}$ for some $\alpha_{0}>0$ which depends on $n, X$ and $Y$.

With the definition of (1.3) in hand, we obtain, when they exist, optimal pointwise upper bounds on $\mathbb{R}^{n} \times(0, \infty)$ for nonnegative solutions $u \in Y$ of the initial value problem (1.1), (1.2) with 
particular emphasis on these bounds as $t \rightarrow 0^{+}$and as $t \rightarrow \infty$. These results are stated in Section 3 and proved in Section 8 .

Since the operator (1.3) is nonlocal, we must require the initial condition (1.2) to hold in $\mathbb{R}^{n} \times(-\infty, 0)$ (not just in $\left.\mathbb{R}^{n} \times\{0\}\right)$ and nonnegative solutions of (1.1), (1.2) may not tend pointwise to zero as $t \rightarrow 0^{+}$(see Theorem 3.5) even though they satisfy the initial condition (1.2).

Of course any estimates we obtain for nonnegative solutions of (1.1), (1.2) also hold for nonnegative solutions of the initial value problem consisting of (1.2) and the equation

$$
\left(\partial_{t}-\Delta\right)^{\alpha} u=u^{\lambda} \quad \text { in } \mathbb{R}^{n} \times \mathbb{R}
$$

According to our results in Section 3 there are essentially only three possibilities for the solutions of (1.1), (1.2) depending on $X, Y, \lambda$, and $\alpha$ :

(i) The only solution is $u \equiv 0$ in $\mathbb{R}^{n} \times \mathbb{R}$;

(ii) There exist sharp nonzero pointwise bounds for solutions as $t \rightarrow 0^{+}$and as $t \rightarrow \infty$;

(iii) There do not exist pointwise bounds for solutions as $t \rightarrow 0^{+}$and as $t \rightarrow \infty$.

All possiblities can occur. For the precise statements of possibilities (i), (ii), and (iii) see Theorem 3.1. Theorems 3.2 3.4, and Theorems 3.5 and 3.6, respectively.

The operator (1.3) is a fully fractional heat operator as opposed to time fractional heat operators in which the fractional derivatives are only with respect to $t$, and space fractional heat operators, in which the fractional derivatives are only with respect to $x$.

Some recent results for nonlinear PDEs containing time (resp. space) fractional heat operators can be found in 2 , 4, 15, 10, 15, 16, 17, 21, 28, 32, 33. (resp. [1, 3, 17, 8, 9, 11, 12, 14, 18, 22, 29, 30, 31]). We know of no results for nonlinear PDEs containing the fully fractional heat operator (1.3). However results for linear PDEs containing (1.3), including in particular

$$
\left(\partial_{t}-\Delta\right)^{\alpha} u=f
$$

where $f$ is a given function, can be found in 6 , 20, 24, 27.

\section{Definition and properties of fully fractional heat operators}

In this section we give a well-motivated definition of the fully fractional heat operator (1.3), suitable for our study of the initial value problem (1.1), (1.2), and then give some of its properties.

Some of the material in this section is inspired by - and can be viewed as the parabolic analog of - the material in [26, Sec. 5.1] concerning the fractional Laplacian.

Since for functions $u: \mathbb{R}^{n} \times \mathbb{R} \rightarrow \mathbb{R}, n \geq 1$, which are sufficiently smooth and small at infinity we have

$$
\left(\left(\partial_{t}-\Delta\right) u\right)^{\wedge}(y, s)=\left(|y|^{2}-i s\right) \widehat{u}(y, s),
$$

where ${ }^{\uparrow}$ is the Fourier transform operator on $\mathbb{R}^{n} \times \mathbb{R}$ given by

$$
\widehat{u}(y, s)=\iint_{\mathbb{R}^{n} \times \mathbb{R}} e^{i(y, s) \cdot(x, t)} u(x, t) d x d t,
$$

the fractional heat operator $\left(\partial_{t}-\Delta\right)^{\alpha}, \alpha>0$, is formally defined in [25, Chapter 2] by

$$
\left(\left(\partial_{t}-\Delta u\right)^{\alpha} u\right)^{\wedge}(y, s)=\left(|y|^{2}-i s\right)^{\alpha} \widehat{u}(y, s) .
$$


If $f=\left(\partial_{t}-\Delta\right)^{\alpha} u$ then from (2.1) and the fact (see [25, Theorem 2.2] and Theorem 2.1(i) below) that

$$
\widehat{\Phi}_{\alpha}(y, s)=\left(|y|^{2}-i s\right)^{-\alpha} \text { for } 0<\alpha<(n+2) / 2
$$

in the sense of tempered distributions where

$$
\Phi_{\alpha}(x, t)=\frac{t^{\alpha-1}}{\Gamma(\alpha)} \frac{1}{(4 \pi t)^{n / 2}} e^{-|x|^{2} /(4 t)} \chi_{(0, \infty)}(t),
$$

we formally get

$$
\widehat{u}=\widehat{\Phi}_{\alpha} \widehat{f}
$$

Hence by the convolution theorem we formally find that

$$
u=J_{\alpha} f:=\Phi_{\alpha} * f
$$

where $*$ is the convolution operation in $\mathbb{R}^{n} \times \mathbb{R}$. Since $\Phi_{\alpha}(x, t)=0$ for $t \leq 0$ we have

$$
J_{\alpha} f(x, t)=\iint_{\mathbb{R}^{n} \times(-\infty, t)} \Phi_{\alpha}(x-\xi, t-\tau) f(\xi, \tau) d \xi d \tau .
$$

By part (ii) of the following theorem, equations (2.1) and (2.3) are equivalent in the sense that

$$
\left(J_{\alpha} f\right)^{\widehat{ }}=\left(|y|^{2}-i s\right)^{-\alpha} \widehat{f} \text { for } f \in L^{1}\left(\mathbb{R}^{n} \times \mathbb{R}\right) \text { and } 0<\alpha<(n+2) / 2
$$

in the sense of tempered distributions.

Theorem 2.1. Suppose $0<\alpha<(n+2) / 2$.

(i) The Fourier transform of $\Phi_{\alpha}(x, t)$ is the function $\left(|y|^{2}-i s\right)^{-\alpha}$ in the sense that

$$
\iint_{\mathbb{R}^{n} \times \mathbb{R}} \Phi_{\alpha}(x, t) \widehat{\varphi}(x, t) d x d t=\iint_{\mathbb{R}^{n} \times \mathbb{R}}\left(|y|^{2}-i s\right)^{-\alpha} \varphi(y, s) d y d s
$$

for all $\varphi \in S$ where $S$ is the Schwarz class of rapidly decreasing functions.

(ii) The identity $\left(J_{\alpha} f\right)^{\wedge}(y, t)=\left(|y|^{2}-i s\right)^{-\alpha} \widehat{f}(y, s)$ holds in the sense that

$$
\iint_{\mathbb{R}^{n} \times \mathbb{R}} J_{\alpha} f(x, t) \widehat{g}(x, t) d x d t=\iint_{\mathbb{R}^{n} \times \mathbb{R}}\left(|y|^{2}-i s\right)^{-\alpha} \widehat{f}(y, s) g(y, s) d y d s
$$

for all $f \in L^{1}\left(\mathbb{R}^{n} \times \mathbb{R}\right)$ and all $g \in S$.

Motivated by these formal calculations, we will now define the operator $\left(\partial_{t}-\Delta\right)^{\alpha}$ as the inverse of a linear operator

$$
J_{\alpha}: X \rightarrow Y
$$

where $J_{\alpha}$ is defined by (2.4) and (2.2) and $X$ and $Y$ are linear spaces whose elements are functions $f: \mathbb{R}^{n} \times \mathbb{R} \rightarrow \mathbb{R}$ such that the operator (2.6) has the following properties:

(P1) it makes sense because the integral in (2.4) defines a real valued measurable function on $\mathbb{R}^{n} \times \mathbb{R}$ for all $f \in X$,

(P2) it is one-to-one and onto, and

(P3) if $u=J_{\alpha} f$ then $f=0$ in $\mathbb{R}^{n} \times(-\infty, 0)$ if and only if $u=0$ in $\mathbb{R}^{n} \times(-\infty, 0)$. 
Property (P3) will be needed to handle the initial condition (1.2). The domain of $J_{\alpha}$ is usually taken to be $L^{p}\left(\mathbb{R}^{n} \times \mathbb{R}\right), 1 \leq p<\frac{n+2}{2 \alpha}$ (see [24, Section 9.2]). However since the region of integration for the integral (2.4) is not $\mathbb{R}^{n} \times \mathbb{R}$ but rather $\mathbb{R}^{n} \times(-\infty, t)$, we see that more natural and less restrictive choices for the domain and range of $J_{\alpha}$ are

$$
\begin{aligned}
X^{p} & :=\bigcap_{T \in \mathbb{R}} L^{p}\left(\mathbb{R}^{n} \times \mathbb{R}_{T}\right) \\
Y_{\alpha}^{p} & :=J_{\alpha}\left(X^{p}\right)
\end{aligned}
$$

respectively, where $\mathbb{R}_{T}=(-\infty, T)$. By (2.7) we mean $X^{p}$ is the set of all measurable functions $f: \mathbb{R}^{n} \times \mathbb{R} \rightarrow \mathbb{R}$ such that

$$
\|f\|_{L^{p}\left(\mathbb{R}^{n} \times \mathbb{R}_{T}\right)}<\infty \quad \text { for all } T \in \mathbb{R} .
$$

The notation in (2.7) should be interpreted similarly elsewhere in this paper.

According to the following two theorems the formal operator

$$
J_{\alpha}: X^{p} \rightarrow Y_{\alpha}^{p}
$$

where $X^{p}$ and $Y_{\alpha}^{p}$ are defined in (2.7) and (2.8), satisfies properties (P1)-(P3) provided either

$$
\left(p>1 \text { and } 0<\alpha<\frac{n+2}{2 p}\right) \quad \text { or } \quad\left(p=1 \text { and } 0<\alpha \leq \frac{n+2}{2 p}\right) .
$$

When $p$ and $\alpha$ satisfy (2.10), part (i) of the following theorem shows that the operator (2.9) satisfies (P1) and parts (ii) and (iii) give some of its properties.

Theorem 2.2. Suppose $p$ and $\alpha$ are real numbers satisfying (2.10) and $f \in X^{p}$. Then

(i) $J_{\alpha} f, J_{\alpha}|f| \in L_{l o c}^{p}\left(\mathbb{R}^{n} \times \mathbb{R}\right)$ and

(ii) $J_{\beta}\left(J_{\gamma} f\right)=J_{\alpha} f$ in $L_{\mathrm{loc}}^{p}\left(\mathbb{R}^{n} \times \mathbb{R}\right)$ whenever $\beta>0, \gamma>0$, and $\beta+\gamma=\alpha$.

If in addition $\alpha>1$ then

(iii) $H J_{\alpha} f=J_{\alpha-1} f$ in $\mathcal{D}^{\prime}\left(\mathbb{R}^{n} \times \mathbb{R}\right)$ where $H=\partial_{t}-\Delta$ is the heat operator.

Remark 2.1. Theorem $2.2(\mathrm{i})$ can be improved to $J_{\alpha} f \in L_{\mathrm{loc}}^{q}\left(\mathbb{R}^{n} \times \mathbb{R}\right)$ when

$$
1<p<\frac{n+2}{2 \alpha} \text { and } \quad \frac{1}{q}=\frac{1}{p}-\frac{2 \alpha}{n+2} .
$$

This can be seen by applying Gopala Rao [13, Theorem 3.1] to the function $f_{T}$ defined in the proof of Theorem 2.2 in Section 6 .

According to the following theorem, if $p$ and $\alpha$ satisfy (2.10) then the operator (2.9) satisfies properties (P2) and (P3) where $X^{p}$ and $Y_{\alpha}^{p}$ are defined by (2.7) and (2.8).

Theorem 2.3. Suppose $p$ and $\alpha$ are real numbers satisfying (2.10). Then

(i) the operator (2.9) is one-to-one and onto, and

(ii) if

$$
f \in X^{p} \text { and } T \in \mathbb{R}
$$

then

$$
\left.f\right|_{\mathbb{R}^{n} \times \mathbb{R}_{T}}=0 \text { if and only if }\left.\left(J_{\alpha} f\right)\right|_{\mathbb{R}^{n} \times \mathbb{R}_{T}}=0 \text {. }
$$


By the results in this section, the following definition is natural and makes sense.

Definition 2.1. Suppose $p$ and $\alpha$ are real numbers satisfying (2.10) and $X^{p}$ and $Y_{\alpha}^{p}$ are defined by (2.7) and (2.8). Then the operator

$$
\left(\partial_{t}-\Delta\right)^{\alpha}: Y_{\alpha}^{p} \rightarrow X^{p}
$$

is defined to be the inverse of the operator (2.9).

Remark 2.2. The functions $\mu_{T}: X^{p} \rightarrow \mathbb{R}, T \in \mathbb{R}$, defined by $\mu_{T}(f)=\|f\|_{L^{p}\left(\mathbb{R}^{n} \times \mathbb{R}_{T}\right)}$, form a separating family of seminorms on $X^{p}$ which turns $X^{p}$ into a locally convex topological vector space (see for example [23, Theorem 1.37]). Thus assuming (2.10) and defining a subset $O^{\prime}$ of $Y_{\alpha}^{p}$ to be open if $O^{\prime}=J_{\alpha}(O)$ for some open set $O \in X^{p}$, we see by Theorem 2.3)(i) that $Y_{\alpha}^{p}$ is also a locally convex topological vector space and the operator (2.12) is a homeomorphism.

We conclude this section by investigating

$$
\lim _{a \rightarrow 0^{+}}\left(\partial_{t}-a^{2} \Delta\right)^{\alpha} \quad \text { and } \quad \lim _{b \rightarrow 0^{+}}\left(b \partial_{t}-\Delta\right)^{\alpha}
$$

where $\alpha>0$.

To do this we first repeat the above procedure with $\partial_{t}-\Delta$ replaced with $b \partial_{t}-a^{2} \Delta$ where $a$ and $b$ are positive constants. The end result after defining

$$
J_{\alpha, a, b}: X^{p} \rightarrow Y_{\alpha, a, b}^{p}:=J_{\alpha, a, b}\left(X^{p}\right)
$$

by

$$
J_{\alpha, a, b} f=\Phi_{\alpha, a, b} * f,
$$

where $a, b, \alpha, p$ are positive constants satisfying (2.10) and

$$
\Phi_{\alpha, a, b}(x, t)=\frac{1}{a^{n} b} \Phi_{\alpha}\left(\frac{x}{a}, \frac{t}{b}\right),
$$

is the following modified version of Definition 2.1.

Definition 2.2. Suppose $a, b, p$ and $\alpha$ are positive constants satsfying (2.10) and $X^{p}$ and $Y_{\alpha, a, b}^{p}$ are defined in (2.7) and (2.13). Then the operator

$$
\left(b \partial_{t}-a^{2} \Delta\right)^{\alpha}: Y_{\alpha, a, b}^{p} \rightarrow X^{p}
$$

is defined to be the inverse of the operator (2.13).

The following theorem states in what sense

$$
\left(\partial_{t}-a^{2} \Delta\right)^{\alpha} \rightarrow \partial_{t}^{\alpha} \quad \text { as } a \rightarrow 0^{+}
$$

where we formally define the equation

$$
\partial_{t}^{\alpha} u=f
$$

to mean

$$
u=J_{\alpha, 0,1} f
$$

where

$$
\left(J_{\alpha, 0,1} f\right)(x, t):=\int_{-\infty}^{t} \frac{(t-\tau)^{\alpha-1}}{\Gamma(\alpha)} f(x, \tau) d \tau
$$

is the Riemann-Liouville integral of $f$ with respect to $t$ of order $\alpha$ with base point $-\infty$. 
Theorem 2.4. Suppose $\alpha>0$ and $f: \mathbb{R}^{n} \times \mathbb{R} \rightarrow \mathbb{R}$ is a continuous function with compact support. Then

$$
J_{\alpha, a, 1} f \rightarrow J_{\alpha, 0,1} f \quad \text { as } a \rightarrow 0^{+}
$$

uniformly on compact subsets of $\mathbb{R}^{n} \times \mathbb{R}$.

The following theorem states in what sense

$$
\left(b \partial_{t}-\Delta\right)^{\alpha} \rightarrow(-\Delta)^{\alpha} \quad \text { as } b \rightarrow 0^{+}
$$

where we formally define the equation

$$
(-\Delta)^{\alpha} u=f
$$

to mean

$$
u=J_{\alpha, 1,0} f
$$

where

$$
\left(J_{\alpha, 1,0} f\right)(x, t):=\frac{1}{\gamma(n, \alpha)} \int_{\mathbb{R}^{n}} \frac{f(y, t) d y}{|x-y|^{n-2 \alpha}}
$$

is the Riesz potential of $f$ with respect to $x$ of order $\alpha$. Here

$$
\gamma(n, \alpha)=\frac{4^{\alpha} \pi^{n / 2} \Gamma(\alpha)}{\Gamma(n / 2-\alpha)}
$$

Theorem 2.5. Suppose $0<2 \alpha<n$ and $f: \mathbb{R}^{n} \times \mathbb{R} \rightarrow \mathbb{R}$ is a continuous function with compact support. Then

$$
J_{\alpha, 1, b} f \rightarrow J_{\alpha, 1,0} f \quad \text { as } b \rightarrow 0^{+}
$$

uniformly on compact subsets of $\mathbb{R}^{n} \times \mathbb{R}$.

\section{Results for fully fractional initial value problems}

In this section we state our results concerning pointwise bounds for nonnegative solutions

$$
u \in Y_{\alpha}^{p}
$$

of the fully fractional initial value problem

$$
\begin{gathered}
0 \leq\left(\partial_{t}-\Delta\right)^{\alpha} u \leq u^{\lambda} \quad \text { in } \mathbb{R}^{n} \times \mathbb{R}, n \geq 1, \\
u=0 \quad \text { in } \mathbb{R}^{n} \times(-\infty, 0)
\end{gathered}
$$

where $\lambda>0$ and, as in the Definition 2.1 of the operator (2.12), $\alpha$ and $p$ satisfy (2.10).

Remark 3.1. If $\alpha$ and $p$ satisfy (2.10) and $u$ satisfies (3.1) and the first inequality in (3.2) then

$$
f:=\left(\partial_{t}-\Delta\right)^{\alpha} u \geq 0 \text { in } \mathbb{R}^{n} \times \mathbb{R}
$$

and hence $u=J_{\alpha} f \geq 0$ in $\mathbb{R}^{n} \times \mathbb{R}$ by (2.4). Thus the assumption that $u$ be nonnegative can be omitted when studying (3.1)-(3.3). 


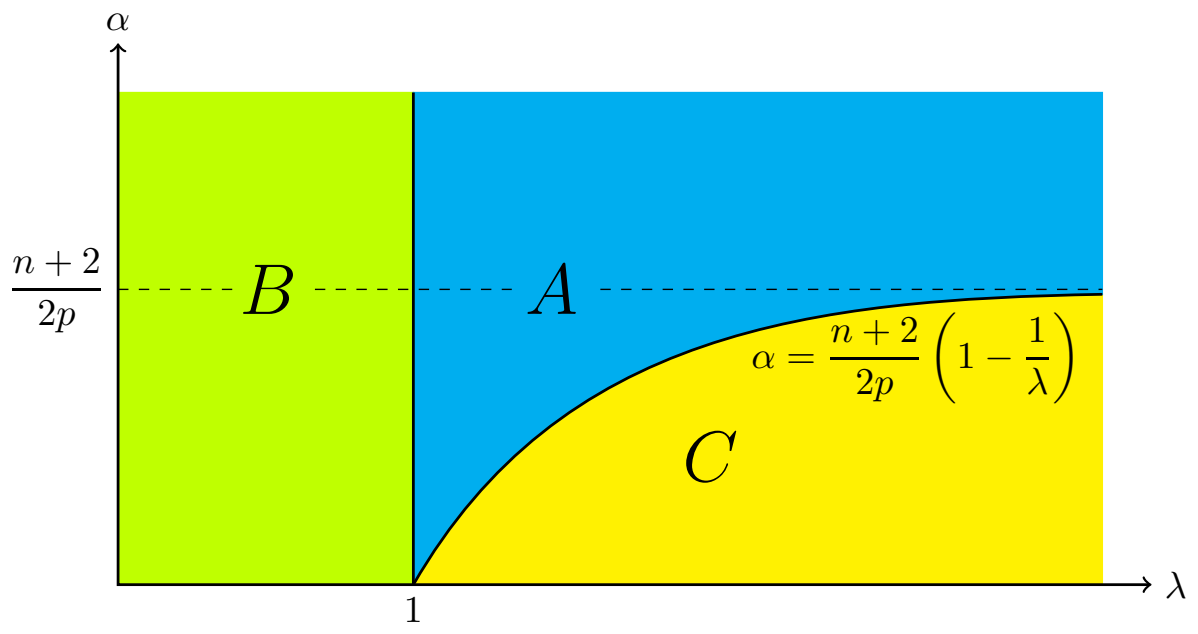

Figure 1: Graphs of the regions $A, B$, and $C$.

In order to state our results we first note that for each fixed $p \geq 1$ the open first quadrant of the $\lambda \alpha$-plane is the union of the following pairwise disjoint sets.

$$
\begin{aligned}
& A=\left\{(\lambda, \alpha): \lambda \geq 1 \text { and } \alpha>\frac{n+2}{2 p}\left(1-\frac{1}{\lambda}\right)\right\} \\
& B=\{(\lambda, \alpha): 0<\lambda<1 \text { and } \alpha>0\} \\
& C=\left\{(\lambda, \alpha): \lambda>1 \text { and } 0<\alpha<\frac{n+2}{2 p}\left(1-\frac{1}{\lambda}\right)\right\} \\
& D=\left\{(\lambda, \alpha): \lambda>1 \text { and } \alpha=\frac{n+2}{2 p}\left(1-\frac{1}{\lambda}\right)\right\} .
\end{aligned}
$$

Note that $A, B$, and $C$ are two dimensional regions in the $\lambda \alpha$-plane whereas $D$ is the curve separating $A$ and $C$. (See Figure 1) Our results in this section deal with solutions of (3.1)-(3.3) when $(\lambda, \alpha)$ is in $A, B$, or $C$. We have no results when $(\lambda, \alpha) \in D$.

The following theorem deals with the case that $(\lambda, \alpha) \in A$.

Theorem 3.1. Suppose $\alpha$ and $p$ satisfy (2.10), $(\lambda, \alpha) \in A$, and $u$ satisfies (3.1) -(3.3). Then

$$
u=\left(\partial_{t}-\Delta\right)^{\alpha} u=0 \quad \text { almost everywhere in } \mathbb{R}^{n} \times \mathbb{R} .
$$

The following three theorems deal with the case $(\lambda, \alpha) \in B$.

Theorem 3.2. Suppose $\alpha$ and $p$ satisfy (2.10), $(\lambda, \alpha) \in B$, and $u$ satisfies (3.1)-(3.3). Then for all $T>0$ we have

$$
\|u\|_{L^{\infty}\left(\mathbb{R}^{n} \times(0, T)\right)} \leq\left(M T^{\alpha}\right)^{\frac{1}{1-\lambda}}
$$

and

$$
\left\|\left(\partial_{t}-\Delta\right)^{\alpha} u\right\|_{L^{\infty}\left(\mathbb{R}^{n} \times(0, T)\right)} \leq\left(M T^{\alpha}\right)^{\frac{\lambda}{1-\lambda}}
$$

where

$$
M=M(\alpha, \lambda)=\frac{\Gamma\left(\frac{\alpha \lambda}{1-\lambda}+1\right)}{\Gamma\left(\alpha+\frac{\alpha \lambda}{1-\lambda}+1\right)}
$$

where $\Gamma$ is the Gamma function. 
By the following theorem the bounds (3.4) and (3.5) in Theorem 3.2 are optimal.

Theorem 3.3. Suppose $\alpha$ and $p$ satisfy (2.10), $(\lambda, \alpha) \in B, T>0$, and $N<M$ where $M$ is given by (3.6). Then there exists a solution

$$
u \in Y_{\alpha}^{p} \cap C\left(\mathbb{R}^{n} \times \mathbb{R}\right)
$$

of (3.2), (3.3) such that

$$
\begin{gathered}
\left(\partial_{t}-\Delta\right)^{\alpha} u \in L^{p}\left(\mathbb{R}^{n} \times \mathbb{R}\right) \cap C\left(\mathbb{R}^{n} \times \mathbb{R}\right), \\
u(0, t) \geq\left(N t^{\alpha}\right)^{\frac{1}{1-\lambda}} \quad \text { for } 0<t<T
\end{gathered}
$$

and

$$
\left(\partial_{t}-\Delta\right)^{\alpha} u(0, t)=\left(N t^{\alpha}\right)^{\frac{\lambda}{1-\lambda}} \quad \text { for } 0<t<T .
$$

Although the estimates (3.4) and (3.5) are optimal there still remains the question as to whether there is a single solution which has the same size as these estimates as $t \rightarrow \infty$. By the following theorem there is such a solution.

Theorem 3.4. Suppose $\alpha$ and $p$ satisfy (2.10) and $(\lambda, \alpha) \in B$. Then there exists $N>0$ and $u \in Y_{\alpha}^{p}$ satisfying (3.2), (3.3) such that

$$
u(x, t) \geq\left(N t^{\alpha}\right)^{\frac{1}{1-\lambda}} \quad \text { for }(x, t) \in \Omega
$$

and

$$
\left(\partial_{t}-\Delta\right)^{\alpha} u(x, t) \geq\left(N t^{\alpha}\right)^{\frac{\lambda}{1-\lambda}} \quad \text { for }(x, t) \in \Omega
$$

where $\Omega=\left\{(x, t) \in \mathbb{R}^{n} \times \mathbb{R}:|x|^{2}<t\right\}$.

According to the following theorem, if $(\lambda, \alpha) \in C$ then there exist bounds as $t \rightarrow 0^{+}$for solutions of (3.1) - (3.3) in neither the pointwise (i.e. $L^{\infty}$ ) sense nor in the $L^{q}$ sense when $q>p$.

Moreover by Theorem [3.6 the same is true as $t \rightarrow \infty$ provided $q \in\left[q_{0}, \infty\right]$ for some $q_{0}=$ $q_{0}(n, \alpha, \lambda)>p$.

Theorem 3.5. Suppose $\alpha$ and $p$ satisfy (2.10)

$$
(\lambda, \alpha) \in C \quad \text { and } \quad q \in(p, \infty] .
$$

Then there exists a solution $u \in Y_{\alpha}^{p}$ of (3.2), (3.3) and a sequence $\left\{t_{j}\right\} \subset(0,1)$ such that

$$
\lim _{j \rightarrow \infty} t_{j}=0
$$

and

where

$$
\left\|u^{\lambda}\right\|_{L^{q}\left(R_{j}\right)}=\left\|\left(\partial_{t}-\Delta\right)^{\alpha} u\right\|_{L^{q}\left(R_{j}\right)}=\infty \quad \text { for } j=1,2, \ldots,
$$

$$
R_{j}=\left\{(x, t) \in \mathbb{R}^{n} \times \mathbb{R}:|x|<\sqrt{t_{j}} \text { and } t_{j}<t<2 t_{j}\right\} .
$$

Theorem 3.6. Suppose $\alpha$ and p satisfy (2.10),

$$
(\lambda, \alpha) \in C \quad \text { and } \quad q \in\left[\frac{n+2}{2 \alpha}\left(1-\frac{1}{\lambda}\right), \infty\right] .
$$

Then there exists a solution $u \in Y_{\alpha}^{p}$ of (3.2), (3.3) and a sequence $\left\{t_{j}\right\} \subset(1, \infty)$ such that

$$
\lim _{j \rightarrow \infty} t_{j}=\infty
$$

and

$$
\left\|u^{\lambda}\right\|_{L^{q}\left(R_{j}\right)}=\left\|\left(\partial_{t}-\Delta\right)^{\alpha} u\right\|_{L^{q}\left(R_{j}\right)}=\infty \quad \text { for } j=1,2, \ldots,
$$

where $R_{j}$ is given in (3.7). 


\section{$4 \quad J_{\alpha}$ version of fully fractional initial value problems}

In order to prove our results stated in Section 3, we will first reformulate them in terms of the inverse $J_{\alpha}$ of the fractional heat operator (2.12) as follows.

Suppose that $\lambda>0$ and, as assumed in Definition 2.1 and Theorems 3.1 3.6, that $p$ and $\alpha$ satisfy (2.10). Then, by Theorem 2.3, $u$ satisfies (3.1)-(3.3) if and only if $f:=\left(\partial_{t}-\Delta\right)^{\alpha} u$ satisfies

$$
\begin{gathered}
f \in X^{p} \\
0 \leq f \leq\left(J_{\alpha} f\right)^{\lambda} \quad \text { in } \mathbb{R}^{n} \times \mathbb{R} \\
f=0 \quad \text { in } \mathbb{R}^{n} \times(-\infty, 0) .
\end{gathered}
$$

Thus the two problems (3.1)-(3.3) and (4.1)-(4.3) are equivalent under the transformation $u=J_{\alpha} f$ when $p$ and $\alpha$ satisfy (2.10). This restriction on $p$ and $\alpha$ was imposed so that $J_{\alpha} f$ would be defined pointwise in $\mathbb{R}^{n} \times \mathbb{R}$ for all $f \in X^{p}$. If $p \geq 1$ and $\alpha>0$ do not satisfy (2.10), that is, if

$$
\left(p>1 \text { and } \alpha \geq \frac{n+2}{2 p}\right) \quad \text { or } \quad\left(p=1 \text { and } \alpha>\frac{n+2}{2 p}\right)
$$

then $J_{\alpha} f$ is generally not defined pointwise as an extended real valued function for $f \in X^{p}$. (However it can be defined for all $f$ in the subspace $L^{p}\left(\mathbb{R}^{n} \times \mathbb{R}\right)$ of $X^{p}$ as a distribution on a certain subspace of the Schwarz space $S$ (see [24, Sec 9.2.5]).

Even though $J_{\alpha} f$ is generally not defined pointwise as and extended real valued function for $f \in X^{p}$ when $p$ and $\alpha$ satisfy (4.4), it is defined pointwise as a nonnegative extended real value function for all nonnegative functions $f \in X^{p}$ for all $p \geq 1$ and $\alpha>0$ because then the integrand of $J_{\alpha} f$ is a nonnegative function. Hence, since $f$ is nonnegative in the problem (4.1)-(4.3), we see that the problem (4.1)-(4.3) makes sense for all $p \geq 1$ and $\alpha>0$ when $J_{\alpha}$ is defined in the pointwise sense, which is the sense in which we will define it in this section. However $J_{\alpha}$, when restricted to the set $X_{+}^{p}$ of all nonnegative functions $f \in X^{p}$, is not one-to-one when $p$ and $\alpha$ satisfy (4.4). Thus our results in this section for the problem (4.1)-(4.3) when $p \geq 1$ and $\alpha>0$ will yield corresponding results for the problem (3.1)-(3.3) only when $p$ and $\alpha$ satisfy (2.10).

In view of these remarks, we will consider in this section solutions

$$
f \in X^{p}
$$

of the following $J_{\alpha}$ version of the fully fractional initial value problem (3.2), (3.3):

$$
\begin{gathered}
0 \leq f \leq K\left(J_{\alpha} f\right)^{\lambda} \quad \text { in } \mathbb{R}^{n} \times \mathbb{R}, n \geq 1 \\
f=0 \quad \text { in } \mathbb{R}^{n} \times(-\infty, 0)
\end{gathered}
$$

where

$$
p \in[1, \infty) \quad \text { and } \quad K, \lambda, \alpha \in(0, \infty)
$$

are constants, $X^{p}$ is defined by (2.7), and $J_{\alpha}$ is given by (2.4) .

Under the equivalence of problems (3.1)-(3.3) and (4.1)-(4.3) discussed above, the following Theorems 4.1 4.6, when restricted to the case that $p$ and $\alpha$ satisfy (2.10) and $K=1$, clearly imply Theorems 3.1 3.6 in Section 3. We will prove Theorems 4.14 4.6 in Section 8 .

Theorem 4.1. Suppose $(\lambda, \alpha) \in A$ and $f, p$, and $K$ satisfy (4.5) (4.8). Then

$$
f=J_{\alpha} f=0 \quad \text { almost everywhere in } \mathbb{R}^{n} \times \mathbb{R} \text {. }
$$


Theorem 4.2. Suppose $(\lambda, \alpha) \in B$ and $f, p$, and $K$ satisfy (4.5)-(4.8). Then for all $b>0$ we have

$$
\|f\|_{L^{\infty}\left(\mathbb{R}^{n} \times(0, b)\right)} \leq K^{\frac{1}{1-\lambda}}\left(M b^{\alpha}\right)^{\frac{\lambda}{1-\lambda}}
$$

and

$$
\left\|J_{\alpha} f\right\|_{L^{\infty}\left(\mathbb{R}^{n} \times(0, b)\right)} \leq K^{\frac{1}{1-\lambda}}\left(M b^{\alpha}\right)^{\frac{1}{1-\lambda}}
$$

where

$$
M=M(\alpha, \lambda)=\frac{\Gamma\left(\frac{\alpha \lambda}{1-\lambda}+1\right)}{\Gamma\left(\alpha+\frac{\alpha \lambda}{1-\lambda}+1\right)} .
$$

Theorem 4.3. Suppose $p$ and $K$ satisfy (4.8), $(\lambda, \alpha) \in B, T>0$, and $0<N<M$ where $M$ is given by (4.12). Then there exists a solution

$$
f \in L^{p}\left(\mathbb{R}^{n} \times \mathbb{R}\right) \cap C\left(\mathbb{R}^{n} \times \mathbb{R}\right)
$$

of (4.6), (4.7) such that

$$
\begin{gathered}
J_{\alpha} f \in C\left(\mathbb{R}^{n} \times \mathbb{R}\right) \\
f(0, t)=K^{\frac{1}{1-\lambda}}\left(N t^{\alpha}\right)^{\frac{\lambda}{1-\lambda}} \quad \text { for } 0<t<T
\end{gathered}
$$

and

$$
J_{\alpha} f(0, t) \geq K^{\frac{1}{1-\lambda}}\left(N t^{\alpha}\right)^{\frac{1}{1-\lambda}} \quad \text { for } 0<t<T .
$$

Theorem 4.4. Suppose $p$ and $K$ satisfy (4.8) and $(\lambda, \alpha) \in B$. Then there exists $N>0$ and

$$
f \in X^{p}
$$

satisfying (4.6), (4.7) such that

$$
f(x, t) \geq K^{\frac{1}{1-\lambda}}\left(N t^{\alpha}\right)^{\frac{\lambda}{1-\lambda}} \quad \text { for }|x|^{2}<t
$$

and

$$
J_{\alpha} f(x, t) \geq K^{\frac{1}{1-\lambda}}\left(N t^{\alpha}\right)^{\frac{1}{1-\lambda}} \text { for }|x|^{2}<t .
$$

Theorem 4.5. Suppose $p$ and $K$ satisfy (4.8),

$$
(\lambda, \alpha) \in C \quad \text { and } \quad q \in(p, \infty] .
$$

Then there exists a solution

$$
f \in L^{p}\left(\mathbb{R}^{n} \times \mathbb{R}\right)
$$

of (4.6), (4.7) and a sequence $\left\{t_{j}\right\} \subset(0,1)$ such that

$$
\lim _{j \rightarrow \infty} t_{j}=0
$$

and

$$
\|f\|_{L^{q}\left(R_{j}\right)}=\infty \quad \text { for } j=1,2, \ldots,
$$

where

$$
R_{j}=\left\{(x, t) \in \mathbb{R}^{n} \times \mathbb{R}:|x|<\sqrt{t_{j}} \text { and } t_{j}<t<2 t_{j}\right\} .
$$


Theorem 4.6. Suppose $p$ and $K$ satisfy (4.8),

$$
(\lambda, \alpha) \in C \quad \text { and } \quad \frac{n+2}{2 \alpha}\left(1-\frac{1}{\lambda}\right) \leq q \leq \infty .
$$

Then there exists a solution

$$
f \in X^{p}
$$

of (4.6), (4.7) and a sequence $\left\{t_{j}\right\} \subset(1, \infty)$ such that

$$
\lim _{j \rightarrow \infty} t_{j}=\infty
$$

and

$$
\|f\|_{L^{q}\left(R_{j}\right)}=\infty \quad \text { for } j=1,2, \ldots,
$$

where $R_{j}$ is given in (4.22).

\section{$5 \quad$ Preliminary results for fully fractional heat operators}

In this section we provide some lemmas needed for the proofs of our results in Section 2 concerning the fully fractional heat operator (2.12).

The following lemma is needed for the proof of Theorem 2.2 .

Lemma 5.1. Suppose $\alpha, \beta>0$. Then

$$
\Phi_{\alpha+\beta}=\Phi_{\alpha} * \Phi_{\beta} \quad \text { in } \mathbb{R}^{n} \times \mathbb{R}
$$

where $\Phi_{\alpha}$ is defined in (2.2).

Proof. Since

$$
\begin{aligned}
& \Phi_{\alpha} * \Phi_{\beta}(x, t)=\int_{-\infty}^{\infty} \int_{\xi \in \mathbb{R}^{n}} \Phi_{\alpha}(x-\xi, t-\tau) \Phi_{\beta}(\xi, \tau) d \xi d \tau \\
& = \begin{cases}0 & \text { for }(x, t) \in \mathbb{R}^{n} \times(-\infty, 0] \\
\int_{0}^{t} \int_{\xi \in \mathbb{R}^{n}} \Phi_{\alpha}(x-\xi, t-\tau) \Phi_{\beta}(\xi, \tau) d \xi d \tau & \text { for }(x, t) \in \mathbb{R}^{n} \times(0, \infty),\end{cases}
\end{aligned}
$$

we have (5.1) holds in $\mathbb{R}^{n} \times(-\infty, 0]$.

Using the well-known facts that

$$
\widehat{\Phi}_{\alpha}(\cdot, t)(y)=\frac{t^{\alpha-1}}{\Gamma(\alpha)} e^{-t|y|^{2}} \quad \text { for } t>0 \text { and } y \in \mathbb{R}^{n}
$$

and

$$
\int_{0}^{t} \frac{(t-\tau)^{\alpha-1} \tau^{\beta-1}}{\Gamma(\alpha) \Gamma(\beta)} d \tau=\frac{t^{\alpha+\beta-1}}{\Gamma(\alpha+\beta)} \quad \text { for } t, \alpha, \beta>0
$$


and assuming we can interchange the order of integration in the following calculation (we will justify this after the calculation) we obtain for $t>0$ and $y \in \mathbb{R}^{n}$ that

$$
\begin{aligned}
& \left(\Phi_{\alpha} * \Phi_{\beta} \widehat{\widehat{r}}(\cdot, t)(y)\right. \\
& =\int_{x \in \mathbb{R}^{n}} e^{i x \cdot y} \int_{0}^{t}\left(\int_{\xi \in \mathbb{R}^{n}} \Phi_{\alpha}(x-\xi, t-\tau) \Phi_{\beta}(\xi, \tau) d \xi\right) d \tau d x \\
& =\int_{0}^{t}\left(\int_{x \in \mathbb{R}^{n}} e^{i x \cdot y}\left(\int_{\xi \in \mathbb{R}^{n}} \Phi_{\alpha}(x-\xi, t-\tau) \Phi_{\beta}(\xi, \tau) d \xi\right) d x\right) d \tau \\
& =\int_{0}^{t} \frac{(t-\tau)^{\alpha-1}}{\Gamma(\alpha)} e^{-|y|^{2}(t-\tau)} \frac{\tau^{\beta-1}}{\Gamma(\beta)} e^{-|y|^{2} \tau} d \tau \quad \text { (by the convolution theorem) } \\
& =e^{-|y|^{2} t} \int_{0}^{t} \frac{(t-\tau)^{\alpha-1} \tau^{\beta-1}}{\Gamma(\alpha) \Gamma(\beta)} d \tau \\
& =e^{-t|y|^{2}} \frac{t^{\alpha+\beta-1}}{\Gamma(\alpha+\beta)}=\widehat{\Phi}_{\alpha+\beta}(\cdot, t)(y)
\end{aligned}
$$

This calculation is justified by Fubini's theorem and the fact that the integral (5.5) with $e^{i x \cdot y}$ replaced with 1 is, by Fubini's theorem for nonnegative functions and (5.4), equal to

$$
\begin{aligned}
& \int_{0}^{t} \int_{\xi \in \mathbb{R}^{n}}\left(\int_{x \in \mathbb{R}^{n}} \Phi_{\alpha}(x-\xi, t-\tau) d x\right) \Phi_{\beta}(\xi, \tau) d \xi d \tau \\
& =\int_{0}^{t} \int_{\xi \in \mathbb{R}^{n}} \frac{(t-\tau)^{\alpha-1}}{\Gamma(\alpha)} \Phi_{\beta}(\xi, \tau) d \xi d \tau \\
& =\frac{t^{\alpha+\beta-1}}{\Gamma(\alpha+\beta)} \text { for } t>0 \text { and } y \in \mathbb{R}^{n}
\end{aligned}
$$

It follows now from (5.6) that (5.1) holds in $\mathbb{R}^{n} \times(0, \infty)$.

The following lemma is needed for the proof of Lemma 5.3 which in turn is needed for the proof of Theorem 2.3 .

Lemma 5.2. Suppose $f \in L^{1}(-\infty, 0)$ and $0<\alpha \leq 1$. Then

$$
g(t):=\int_{-\infty}^{t}(t-\tau)^{\alpha-1}|f(\tau)| d \tau<\infty \quad \text { for almost all } t \in(-\infty, 0) .
$$

Proof. The lemma is clearly true if $\alpha=1$. Hence we can assume $0<\alpha<1$. Since

$$
\begin{aligned}
\int_{-\infty}^{0} & (-t)^{-\alpha} g(t) d t=\int_{-\infty}^{0}(-t)^{-\alpha} \int_{-\infty}^{t}(t-\tau)^{\alpha-1}|f(\tau)| d \tau d t \\
& =\int_{-\infty}^{0}|f(\tau)|\left(\int_{\tau}^{0}(-t)^{(1-\alpha)-1}(t-\tau)^{\alpha-1} d t\right) d \tau \\
& =\Gamma(1-\alpha) \Gamma(\alpha) \int_{-\infty}^{0}|f(\tau)| d \tau<\infty
\end{aligned}
$$

where we have used (5.4), we see that $g(t)<\infty$ for almost all $t \in(-\infty, 0)$. 
Lemma 5.3. Suppose $f \in L^{1}\left(\mathbb{R}^{n} \times(-\infty, 0)\right), \alpha \in(0,1]$, and $y \in \mathbb{R}^{n}$. Then for almost all $t \in$ $(-\infty, 0)$ we have

$$
\widehat{J_{\alpha} f}(\cdot, t)(y)=\int_{-\infty}^{t} \frac{(t-\tau)^{\alpha-1}}{\Gamma(\alpha)} e^{-|y|^{2}(t-\tau)} \widehat{f}(\cdot, \tau)(y) d \tau .
$$

Proof. By Fubini's theorem for nonnegative functions and Lemma 5.2 we find for almost all $t \in$ $(-\infty, 0)$ that

$$
\begin{gathered}
\int_{x \in \mathbb{R}^{n}}\left|e^{i x \cdot y}\right| \int_{-\infty}^{t} \frac{(t-\tau)^{\alpha-1}}{\Gamma(\alpha)} \int_{\xi \in \mathbb{R}^{n}} \Phi_{1}(x-\xi, t-\tau)|f(\xi, \tau)| d \xi d \tau d x \\
=\int_{-\infty}^{t} \frac{(t-\tau)^{\alpha-1}}{\Gamma(\alpha)}\left(\int_{\xi \in \mathbb{R}^{n}}|f(\xi, \tau)| d \xi\right) d \tau<\infty .
\end{gathered}
$$

Hence by Fubini's theorem, the convolution theorem for Fourier transforms, and (5.3), we see for almost all $t \in(-\infty, 0)$ that

$$
\begin{aligned}
\widehat{J_{\alpha} f}(\cdot, t)(y) & =\int_{-\infty}^{t} \int_{x \in \mathbb{R}^{n}} e^{i x \cdot y} \int_{\xi \in \mathbb{R}^{n}} \Phi_{\alpha}(x-\xi, t-\tau) f(\xi, \tau) d \xi d x d \tau \\
& =\int_{-\infty}^{t} \frac{(t-\tau)^{\alpha-1}}{\Gamma(\alpha)} e^{-|y|^{2}(t-\tau)} \widehat{f}(\cdot, \tau)(y) d \tau
\end{aligned}
$$

\section{$6 \quad$ Fully fractional heat operator proofs}

In this section we prove our fully fractional heat operator results which we stated in Section 2 ,

Proof of Theorem 2.1. Part (i) was proved by Sampson [25, Theorem 2.2]. We prove part (ii) in two steps.

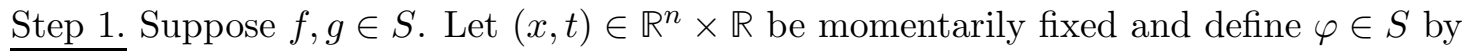

$$
\varphi(y, s)=f(x+y, t+s) .
$$

Then

$$
\widehat{\widehat{\varphi}}(y, s)=(2 \pi)^{n+1} \varphi(-y,-s)=(2 \pi)^{n+1} f(x-y, t-s)
$$

and

$$
\widehat{\varphi}(y, s)=e^{-i x \cdot y-i t s} \widehat{f}(y, s) .
$$

Thus by part (i) with $\varphi$ replaced with $\widehat{\varphi}$ we get

$$
\begin{aligned}
(2 \pi)^{n+1} J_{\alpha} f(x, t) & =(2 \pi)^{n+1} \iint_{\mathbb{R}^{n} \times \mathbb{R}} \Phi_{\alpha}(y, s) f(x-y, t-s) d y d s \\
& =\iint_{\mathbb{R}^{n} \times \mathbb{R}} \Phi_{\alpha}(y, s) \widehat{\hat{\varphi}}(y, s) d y d s \\
& =\iint_{\mathbb{R}^{n} \times \mathbb{R}}\left(|y|^{2}-i s\right)^{-\alpha} \widehat{\varphi}(y, s) d y d s \\
& =\iint_{\mathbb{R}^{n} \times \mathbb{R}}\left(|y|^{2}-i s\right)^{-\alpha} \widehat{f}(y, s) e^{-i x \cdot y-i t s} d y d s .
\end{aligned}
$$


Multiplying (6.1) by $\widehat{g}(x, t) /(2 \pi)^{n+1}$, integrating the resulting equation with respect to $(x, t)$, and interchanging the order of integration in the resulting integral on the RHS, which is allowed by Fubini's theorem and the fact that

$$
\left.\iint_{\left.|| y\right|^{2}-i s \mid \leq 1}|| y\right|^{2}-\left.i s\right|^{-\alpha} d y d s<\infty \quad \text { for } 0<\alpha<(n+2) / 2
$$

we get (2.5).

Step 2. Suppose $f \in L^{1}\left(\mathbb{R}^{n} \times \mathbb{R}\right)$ and $g \in S$. Then $\widehat{g} \in S$ and $\widehat{f} \in C\left(\mathbb{R}^{n} \times \mathbb{R}\right) \cap L^{\infty}\left(\mathbb{R}^{n} \times \mathbb{R}\right)$. Since $\bar{S}$ is dense in $L^{1}\left(\mathbb{R}^{n} \times \mathbb{R}\right)$ there exists $\left\{f_{j}\right\} \subset S$ such that $f_{j} \rightarrow f$ in $L^{1}\left(\mathbb{R}^{n} \times \mathbb{R}\right)$ and by Step 1

$$
\iint_{\mathbb{R}^{n} \times \mathbb{R}} J_{\alpha} f_{j}(x, t) \widehat{g}(x, t) d x d t=\iint_{\mathbb{R}^{n} \times \mathbb{R}}\left(|y|^{2}-i s\right)^{-\alpha} \widehat{f}_{j}(y, s) g(y, s) d y d s .
$$

Since

$$
\left\|\widehat{f}_{j}-\widehat{f}\right\|_{L^{\infty}\left(\mathbb{R}^{n} \times \mathbb{R}\right)} \leq\left\|f_{j}-f\right\|_{L^{1}\left(\mathbb{R}^{n} \times \mathbb{R}\right)} \rightarrow 0 \quad \text { as } j \rightarrow \infty
$$

we have

$$
\begin{aligned}
& \left|\iint_{\mathbb{R}^{n} \times \mathbb{R}}\left(\widehat{f}_{j}(y, s)-\widehat{f}(y, s)\right)\left(|y|^{2}-i s\right)^{-\alpha} g(y, s) d y d s\right| \\
& \quad \leq\left.\left\|\widehat{f}_{j}-\widehat{f}\right\|_{L^{\infty}\left(\mathbb{R}^{n} \times \mathbb{R}\right)} \iint_{\mathbb{R}^{n} \times \mathbb{R}}|| y\right|^{2}-\left.i s\right|^{-\alpha}|g(y, s)| d y d s \\
& \quad \rightarrow 0 \text { as } j \rightarrow \infty
\end{aligned}
$$

by (6.2). Thus the RHS of (6.3) tends to the RHS of (2.5) as $j \rightarrow \infty$.

Also, defining $h(x, t)=|\widehat{g}(-x,-t)|$ we have

$$
\begin{aligned}
\mid \iint_{\mathbb{R}^{n} \times \mathbb{R}} J_{\alpha}\left(f_{j}\right. & -f)(x, t) \widehat{g}(x, t) d x d t \mid \\
& \leq \iint_{\mathbb{R}^{n} \times \mathbb{R}} \iint_{\mathbb{R}^{n} \times \mathbb{R}} \Phi_{\alpha}(x-y, t-s)\left|\left(f_{j}-f\right)(y, s)\right| d y d s|\widehat{g}(x, t)| d x d t \\
& =\iint_{\mathbb{R}^{n} \times \mathbb{R}}\left|\left(f_{j}-f\right)(y, s)\right|\left(\Phi_{\alpha} * h\right)(-y,-s) d y d s \\
& \rightarrow 0 \quad \text { as } j \rightarrow \infty
\end{aligned}
$$

because noting that $h \in L^{1}\left(\mathbb{R}^{n} \times \mathbb{R}\right) \cap L^{\infty}\left(\mathbb{R}^{n} \times \mathbb{R}\right)$,

$$
\begin{aligned}
\left\|\Phi_{\alpha} \chi_{\mathbb{R}^{n} \times(0,1)}\right\|_{L^{1}\left(\mathbb{R}^{n} \times \mathbb{R}\right)} & =\int_{0}^{1} \frac{t^{\alpha-1}}{\Gamma(\alpha)} \int_{x \in \mathbb{R}^{n}} \Phi_{1}(x, t) d x d t \\
& =\int_{0}^{1} \frac{t^{\alpha-1}}{\Gamma(\alpha)} d t<\infty \text { for } \alpha>0
\end{aligned}
$$

and $\Phi_{\alpha} \chi_{\mathbb{R}^{n} \times(1, \infty)} \in L^{\infty}\left(\mathbb{R}^{n} \times \mathbb{R}\right)$ for $\alpha<(n+2) / 2$ we find that

$$
\Phi_{\alpha} * h=\Phi_{\alpha} \chi_{\mathbb{R}^{n} \times(0,1)} * h+\Phi_{\alpha} \chi_{\mathbb{R}^{n} \times(1, \infty)} * h \in L^{\infty}\left(\mathbb{R}^{n} \times \mathbb{R}\right)
$$

by Young's inequality. Thus the LHS of (6.3) tends to the LHS of (2.5) as $j \rightarrow \infty$. 
Proof of Theorem 2.2. Since

$$
\bigcap_{T \in \mathbb{R}} L_{\mathrm{loc}}^{p}\left(\mathbb{R}^{n} \times \mathbb{R}_{T}\right)=L_{\mathrm{loc}}^{p}\left(\mathbb{R}^{n} \times \mathbb{R}\right)
$$

and since $\left.\left(J_{\alpha} f_{T}\right)\right|_{\mathbb{R}^{n} \times \mathbb{R}_{T}}=\left.\left(J_{\alpha} f\right)\right|_{\mathbb{R}^{n} \times \mathbb{R}_{T}}$, where $f_{T}=f \chi_{\mathbb{R}^{n} \times \mathbb{R}_{T}}$ to prove (i), (ii) and (iii) it suffices to prove for all $T \in \mathbb{R}$ that

$(\mathrm{i})^{\prime} \quad J_{\alpha} f_{T}, J_{\alpha}\left|f_{T}\right| \in L_{\mathrm{loc}}^{p}\left(\mathbb{R}^{n} \times \mathbb{R}_{T}\right)$

$(\text { ii })^{\prime} \quad J_{\beta} J_{\gamma} f_{T}=J_{\alpha} f_{T} \quad$ in $L_{\text {loc }}^{p}\left(\mathbb{R}^{n} \times \mathbb{R}_{T}\right)$ whenever $\beta>0, \gamma>0$, and $\beta+\gamma=\alpha$ and

(iii) $H J_{\alpha} f_{T}=J_{\alpha-1} f_{T}$ in $D^{\prime}\left(\mathbb{R}^{n} \times \mathbb{R}_{T}\right)$ when $\alpha>1$.

To do this, let $T \in \mathbb{R}$ be fixed. Since $f \in X^{p} \subset L^{p}\left(\mathbb{R}^{n} \times \mathbb{R}_{T}\right)$ we have

$$
f_{T} \in L^{p}\left(\mathbb{R}^{n} \times \mathbb{R}\right) .
$$

Proof of (i)'. Since $\left|J_{\alpha} f_{T}\right| \leq J_{\alpha}\left|f_{T}\right|$, to prove (i)' it suffices to prove only that

$$
J_{\alpha}\left|f_{T}\right| \in L_{\mathrm{loc}}^{p}\left(\mathbb{R}^{n} \times \mathbb{R}_{T}\right) .
$$

By (2.3) we have

$$
J_{\alpha}\left|f_{T}\right|=u_{1}+u_{2}
$$

where

$$
u_{1}=\left(\Phi_{\alpha} \chi_{\mathbb{R}^{n} \times(0,1)}\right) *\left|f_{T}\right| \quad \text { and } \quad u_{2}=\left(\Phi_{\alpha} \chi_{\mathbb{R}^{n} \times(1, \infty)}\right) *\left|f_{T}\right| .
$$

It follows from (6.4), (6.5), and Young's inequality that

$$
u_{1} \in L^{p}\left(\mathbb{R}^{n} \times \mathbb{R}\right) .
$$

Thus to complete the proof of (6.6) and hence of (i)' it suffices to show

$$
u_{2} \in L^{\infty}\left(\mathbb{R}^{n} \times \mathbb{R}\right) .
$$

To do this we consider two cases.

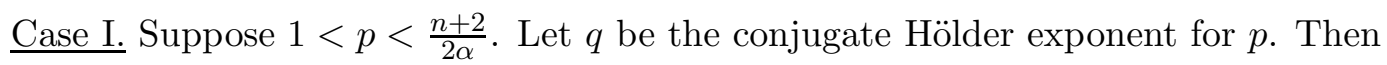

$$
\frac{1}{q}=1-\frac{1}{p}<1-\frac{2 \alpha}{n+2}=\frac{n+2-2 \alpha}{n+2}
$$

and thus making the change of variables $\sqrt{\frac{q}{4 s}} y=z$ we obtain

$$
\begin{array}{r}
\left\|\Phi_{\alpha} \chi_{\mathbb{R}^{n} \times(1, \infty)}\right\|_{L^{q}\left(\mathbb{R}^{n} \times \mathbb{R}\right)}^{q}=C(n, \alpha, q) \int_{1}^{\infty} \int_{y \in \mathbb{R}^{n}} s^{(\alpha-1-n / 2) q} e^{-\frac{q}{4 s}|y|^{2}} d y d s \\
=C(n, \alpha, q) \int_{1}^{\infty} s^{(\alpha-1-n / 2) q+n / 2} \int_{z \in \mathbb{R}^{n}} e^{-|z|^{2}} d z d s<\infty .
\end{array}
$$

Hence (6.8) follows from (6.5) and Young's inequality. 
Case II. Suppose $1=p \leq \frac{n+2}{2 \alpha}$. Then

$$
\begin{aligned}
\Phi_{\alpha} \chi_{\mathbb{R}^{n} \times(1, \infty)}(y, s) & \leq C(n, \alpha) s^{\alpha-1-n / 2} \chi_{\mathbb{R}^{n} \times(1, \infty)}(y, s) \\
& \leq C(n, \alpha) \quad \text { for }(y, s) \in \mathbb{R}^{n} \times \mathbb{R} .
\end{aligned}
$$

Thus (6.8) follows from (6.5) and so the proof of (i)' is complete.

Proof of (ii)'. Using Fubini's theorem for nonnegative functions and Lemma 5.1 we have

$$
\begin{aligned}
J_{\beta}\left(J_{\gamma}\left|f_{T}\right|\right)(x, t) & =\iint_{\mathbb{R}^{n} \times \mathbb{R}} \Phi_{\beta}(x-\xi, t-\tau) \iint_{\mathbb{R}^{n} \times \mathbb{R}} \Phi_{\gamma}(\xi-\eta, \tau-\zeta)\left|f_{T}(\eta, \zeta)\right| d \eta d \zeta d \xi d \tau \\
& =\iint_{\mathbb{R}^{n} \times \mathbb{R}} \Phi_{\beta+\gamma}(x-\eta, t-\zeta)\left|f_{T}(\eta, \zeta)\right| d \eta d \zeta \\
& =\left(J_{\alpha}\left|f_{T}\right|\right)(x, t)<\infty \quad \text { a.e. in } \mathbb{R}^{n} \times \mathbb{R}
\end{aligned}
$$

by part (i)'. Hence by Fubini's theorem the above calculation can be repeated with $\left|f_{T}\right|$ replaced with $f_{T}$ which gives (ii)'.

Proof of (iii)'. By (i)' we have

$$
J_{\alpha}\left|f_{T}\right|, J_{\alpha-1}\left|f_{T}\right| \in L_{\mathrm{loc}}^{p}\left(\mathbb{R}^{n} \times \mathbb{R}_{T}\right) \subset D^{\prime}\left(\mathbb{R}^{n} \times \mathbb{R}_{T}\right) .
$$

Let $\varphi \in C_{0}^{\infty}\left(\mathbb{R}^{n} \times \mathbb{R}_{T}\right)$. Then noting that

$$
\iint_{\mathbb{R}^{n} \times \mathbb{R}_{T}} \Phi_{1}(x-\eta, t-\zeta) H^{*} \varphi(x, t) d x d t=\varphi(\eta, \zeta) \quad \text { for }(\eta, \zeta) \in \mathbb{R}^{n} \times \mathbb{R}_{T}
$$

where $H^{*}=-\partial_{t}-\Delta$ and assuming we can interchange the order of integration in the following calculation (we will justify this after the calculation) it follows from Lemma 5.1 that

$$
\begin{aligned}
& \left(H\left(J_{\alpha} f_{T}\right)\right)(\varphi)=\left(J_{\alpha} f_{T}\right)\left(H^{*} \varphi\right) \\
& =\iint_{\mathbb{R}^{n} \times \mathbb{R}_{T}}\left(\iint_{\mathbb{R}^{n} \times \mathbb{R}_{T}} \Phi_{\alpha}(x-\xi, t-\tau) f_{T}(\xi, \tau) d \xi d \tau\right) H^{*} \varphi(x, t) d x d t \\
& =\iint_{\mathbb{R}^{n} \times \mathbb{R}_{T}} \quad \iint_{\mathbb{R}^{n} \times \mathbb{R}_{T}}\left(\iint_{\mathbb{R}^{n} \times \mathbb{R}_{T}} \Phi_{1}(x-\eta, t-\zeta) \Phi_{\alpha-1}(\eta-\xi, \zeta-\tau) d \eta d \zeta\right) \\
& \quad \times f_{T}(\xi, \tau) d \xi d \tau H^{*} \varphi(x, t) d x d t \\
& =\iint_{\mathbb{R}^{n} \times \mathbb{R}_{T}}\left(\iint_{\mathbb{R}^{n} \times \mathbb{R}_{T}}\left(\iint_{\mathbb{R}^{n} \times \mathbb{R}_{T}} \Phi_{1}(x-\eta, t-\zeta) H^{*} \varphi(x, t) d x d t\right) \Phi_{\alpha-1}(\eta-\xi, \zeta-\tau) d \eta d \zeta\right) \\
& \quad \times f_{T}(\xi, \tau) d \xi d \tau \\
& =\iint_{\mathbb{R}^{n} \times \mathbb{R}_{T}}\left(\iint_{\mathbb{R}^{n} \times \mathbb{R}_{T}} \Phi_{\alpha-1}(\eta-\xi, \zeta-\tau) f_{T}(\xi, \tau) d \xi d \tau\right) \varphi(\eta, \zeta) d \eta d \zeta \\
& =\left(J_{\alpha-1} f_{T}\right)(\varphi) .
\end{aligned}
$$


To justify this calculation, it suffices by Fubini's theorem to show the integral (6.12), with $f_{T}$ and $H^{*} \varphi$ replaced with $\left|f_{T}\right|$ and $\left|H^{*} \varphi\right|$, is finite. However in the same way that (6.12) was obtained from (6.11), we see that this modified integral equals

$$
\iint_{\mathbb{R}^{n} \times \mathbb{R}_{T}}\left(J_{\alpha}\left|f_{T}\right|\right)(x, t)\left|H^{*} \varphi\right|(x, t) d x d t<\infty
$$

by (6.9).

Proof of Theorem [2.3. Clearly (ii) implies (i). We now prove (ii). Suppose (2.11). It follows from (2.4) that

$$
\left.f\right|_{\mathbb{R}^{n} \times \mathbb{R}_{T}}=0 \text { implies }\left.\left(J_{\alpha} f\right)\right|_{\mathbb{R}^{n} \times \mathbb{R}_{T}}=0 .
$$

Conversely suppose

$$
\left.\left(J_{\alpha} f\right)\right|_{\mathbb{R}^{n} \times \mathbb{R}_{T}}=0 .
$$

The complete the proof of (ii) it suffices to prove

$$
\left.f\right|_{\mathbb{R}^{n} \times \mathbb{R}_{T}}=0 .
$$

By Theorem 2.2(iii) and mathematical induction, we can, without loss of generality, assume for the proof (6.14) that

$$
0<\alpha \leq 1
$$

Moreover, by translating we can assume

$$
T=0
$$

We divide the proof of (6.14) into two cases.

Case I. Suppose $(\underline{2.10})_{2}$ holds. Then

$$
1=p \leq \frac{n+1}{2 \alpha}
$$

Let

$$
F(y, t)=\widehat{f}(\cdot, t)(y) \quad \text { for }(y, t) \in \mathbb{R}^{n} \times(-\infty, 0) .
$$

By (2.11) and (6.17) we have

$$
f \in L^{1}\left(\mathbb{R}^{n} \times(-\infty, 0)\right)
$$

and thus

$$
f(\cdot, t) \in L^{1}\left(\mathbb{R}^{n}\right) \quad \text { for almost all } t \in(-\infty, 0)
$$

which implies

$$
F(\cdot, t) \in C\left(\mathbb{R}^{n}\right) \quad \text { for almost all } t \in(-\infty, 0) .
$$

Also, by (6.19)

$$
\begin{aligned}
\|F(y, \cdot)\|_{L^{1}(-\infty, 0)} & =\int_{-\infty}^{0}\left|\int_{\mathbb{R}^{n}} e^{i x \cdot y} f(x, t) d x\right| d t \\
& \leq\|f\|_{L^{1}\left(\mathbb{R}^{n} \times(-\infty, 0)\right.}<\infty \text { for all } y \in \mathbb{R}^{n}
\end{aligned}
$$


Case I(a). Suppose $\alpha=1$. Then by (6.19), (6.13), and Lemma 5.3 we have for each $y \in \mathbb{R}^{n}$ that

$$
\int_{-\infty}^{t} e^{|y|^{2} \tau} F(y, \tau) d \tau=e^{|y|^{2} t} \int_{-\infty}^{t} e^{-|y|^{2}(t-\tau)} F(y, \tau) d \tau=0
$$

for almost all $t \in(-\infty, 0)$. Hence, by (6.20) and the measure theoretic fundamental theorem of calculus, we get $F=0$ in $L^{1}\left(\mathbb{R}^{n} \times(-\infty, 0)\right)$ which together with (6.18) implies (6.14).

Case $\mathrm{I}(\mathrm{b})$. Suppose $0<\alpha<1$. To handle this case we hold $y \in \mathbb{R}^{n} \backslash\{0\}$ fixed and define

$$
F_{0}(t):=F(y, t)
$$

Then by (6.20)

$$
F_{0} \in L^{1}(-\infty, 0)
$$

From (6.19), (6.13), and Lemma 5.3 we have

$$
g(t):=\int_{-\infty}^{t}(t-\tau)^{\alpha-1} e^{|y|^{2} \tau} F_{0}(\tau) d \tau=0
$$

for almost all $t \in(-\infty, 0)$. On the other hand, assuming we can interchange the order of integration in the following calculation (we will justify this after the calculation), we find for $b \in \mathbb{R}$ that

$$
\begin{aligned}
& \int_{-\infty}^{0}\left(\int_{t}^{0}(\zeta-t)^{-\alpha} \cos b \zeta d \zeta\right) g(t) d t \\
& =\int_{-\infty}^{0} e^{|y|^{2} \tau} F_{0}(\tau)\left(\int_{\tau}^{0} \cos b \zeta\left(\int_{\tau}^{\zeta}(t-\tau)^{\alpha-1}(\zeta-t)^{-\alpha} d t\right) d \zeta\right) d \tau \\
& =C(\alpha) \int_{-\infty}^{0} e^{|y|^{2} \tau} F_{0}(\tau)\left(\int_{\tau}^{0} \cos b \zeta d \zeta\right) d \tau
\end{aligned}
$$

because making the change of variables $t=\zeta-(\zeta-\tau) s$ we see that

$$
\int_{\tau}^{\zeta}(t-\tau)^{\alpha-1}(\zeta-t)^{-\alpha} d t=\int_{0}^{1}(1-s)^{\alpha-1} s^{-\alpha} d s=C(\alpha)
$$

The calculation (6.24) is justified by Fubini's theorem and the fact that if we replace $\cos b \zeta$ and $g(t)$ with $|\cos b \zeta|$ and

$$
g_{0}(t)=\int_{-\infty}^{t}(t-\tau)^{\alpha-1} e^{|y|^{2} \tau}\left|F_{0}(\tau)\right| d \tau
$$

respectively in the above calculation we get by Fubini's theorem for nonnegative functions that

$$
\begin{aligned}
& \int_{-\infty}^{0} \int_{t}^{0}(\zeta-t)^{-\alpha}|\cos b \zeta| d \zeta g_{0}(t) d t \\
& \leq C(\alpha) \int_{-\infty}^{0} e^{|y|^{2} \tau}\left|F_{0}(\tau)\right|\left(\int_{\tau}^{0}|\cos b \zeta| d \zeta\right) d \tau \\
& \leq C(\alpha) \int_{-\infty}^{0}(-\tau) e^{|y|^{2} \tau}\left|F_{0}(\tau)\right| d \tau<\infty
\end{aligned}
$$

by 6.22 
It follows now from (6.23), (6.24) and (6.21) that

$$
0=\int_{-\infty}^{0} e^{|y|^{2} \tau} F(y, \tau) \sin b \tau d \tau
$$

for all $y \in \mathbb{R}^{n} \backslash\{0\}$ and all $b \in \mathbb{R}$. Thus since the Fourier sine transform is one to one on $L^{1}(-\infty, 0)$ we have $F(y, \cdot)=0$ in $L^{1}(-\infty, 0)$ for all $y \in \mathbb{R}^{n} \backslash\{0\}$. Hence by Fubini's theorem, $F=0$ in $L^{1}\left(\mathbb{R}^{n} \times(-\infty, 0)\right)$, which together with (6.18) and (6.16) implies (6.14).

Case II. Suppose (2.10) 1 holds. Let $f_{T}=f \chi_{\mathbb{R}^{n} \times \mathbb{R}_{T}}$ and $u=J_{\alpha} f_{T}$. Then by (2.11) we have

$$
f_{T} \in L^{p}\left(\mathbb{R}^{n} \times \mathbb{R}\right),
$$

and by (2.4) and (6.13) we have

$$
u=0 \quad \text { in } \mathbb{R}^{n} \times \mathbb{R}_{T} .
$$

Let $J_{\varepsilon}^{-\alpha} u$ be as defined in Theorem A.1. By (6.25) we have for $l>\alpha$ that $\left(\Delta_{y, \tau}^{l} u\right)(x, t)=0$ for $(x, t) \in \mathbb{R}^{n} \times \mathbb{R}_{T}$ and $(y, \tau) \in \mathbb{R}^{n} \times(0, \infty)$. Thus for $\varepsilon>0$ we have

$$
J_{\varepsilon}^{-\alpha} u=0 \quad \text { in } \mathbb{R}^{n} \times \mathbb{R}_{T} .
$$

Hence (6.14) follows from Theorem A.1.

Proof of Theorem 2.4. For $a, \tau>0$ and $\delta \geq 0$ we have

$$
\begin{aligned}
\int_{|\xi|>\delta} \Phi_{\alpha, a, 1}(\xi, \tau) d \xi & =\int_{|\xi|>\delta} \frac{\tau^{\alpha-1}}{\Gamma(\alpha)} \frac{1}{\left(4 \pi a^{2} \tau\right)^{n / 2}} e^{-\frac{|\xi|^{2}}{4 a^{2} \tau}} d \xi \\
& =\frac{\tau^{\alpha-1}}{\Gamma(\alpha)} \frac{1}{\pi^{n / 2}} \int_{|\eta|>\frac{\delta}{\sqrt{4 a^{2} \tau}}} e^{-|\eta|^{2}} d \eta .
\end{aligned}
$$

In particular, taking $\delta=0$ we find that

$$
\int_{\mathbb{R}^{n}} \Phi_{\alpha, a, 1}(\xi, \tau) d \xi=\frac{\tau^{\alpha-1}}{\Gamma(\alpha)} \quad \text { for } a, \tau>0
$$

Let $\Omega$ be a compact subset of $\mathbb{R}^{n} \times \mathbb{R}$. Choose $T>0$ such that

$$
f=0 \quad \text { on } \mathbb{R}^{n} \times \mathbb{R}_{-T}
$$

and

$$
\Omega \subset \mathbb{R}^{n} \times \mathbb{R}_{T} .
$$

Let $\varepsilon>0$. Since $f$ is uniformly continuous on $\mathbb{R}^{n} \times \mathbb{R}$ there exists $\delta>0$ such that

$$
|f(x-\xi, \zeta)-f(x, \zeta)|<\varepsilon
$$

whenever $x, \xi \in \mathbb{R}^{n}, \zeta \in \mathbb{R}$, and $|\xi|<\delta$.

Let $(x, t) \in \Omega$. Then $t<T$ and thus for $\tau \geq 2 T$ we have

$$
t-\tau<T-2 T=-T
$$


Hence for $a>0$ we have by (6.28) and (6.27) that

$$
\begin{aligned}
& \left|\left(J_{\alpha, a, 1} f-J_{\alpha, 0,1} f\right)(x, t)\right| \\
& \leq \int_{0}^{2 T}\left|\int_{\mathbb{R}^{n}} \Phi_{\alpha, a, 1}(\xi, \tau) f(x-\xi, t-\tau) d \xi-\frac{\tau^{\alpha-1}}{\Gamma(\alpha)} f(x, t-\tau)\right| d \tau \\
& =\int_{0}^{2 T}\left|\int_{\mathbb{R}^{n}} \Phi_{\alpha, a, 1}(\xi, \tau)(f(x-\xi, t-\tau)-f(x, t-\tau)) d \xi\right| d \tau \\
& \leq K_{1}(x, t)+K_{2}(x, t)
\end{aligned}
$$

where

$$
K_{1}(x, t)=\int_{0}^{2 T} \int_{|\xi|<\delta} \Phi_{\alpha, a, 1}(\xi, \tau)|f(x-\xi, t-\tau)-f(x, t-\tau)| d \xi d \tau
$$

and

$$
K_{2}(x, t)=\int_{0}^{2 T} \int_{|\xi|>\delta} \Phi_{\alpha, a, 1}(\xi, \tau)|f(x-\xi, t-\tau)-f(x, t-\tau)| d \xi d \tau .
$$

From (6.30) and (6.27) we conclude that

$$
K_{1}(x, t) \leq \varepsilon \int_{0}^{2 T}\left(\int_{\mathbb{R}^{n}} \Phi_{\alpha, a, 1}(\xi, \tau) d \xi\right) d \tau=\varepsilon \int_{0}^{2 T} \frac{\tau^{\alpha-1}}{\Gamma(\alpha)} d \tau
$$

and letting $M=2\|f\|_{L^{\infty}\left(\mathbb{R}^{n} \times \mathbb{R}\right)}$ and using (6.26) we obtain

$$
K_{2}(x, t) \leq M \int_{0}^{2 T}\left(\int_{|\xi|>\delta} \Phi_{\alpha, a, 1}(\xi, \tau) d \xi\right) d \tau \leq M\left(\int_{0}^{2 T} \frac{\tau^{\alpha-1}}{\Gamma(\alpha)} d \tau\right) C(n, a, \delta, T)
$$

where

$$
C(n, a, \delta, T)=\frac{1}{\pi^{n / 2}} \int_{|\eta|>\frac{\delta}{\sqrt{8 a^{2} T}}} e^{-|\eta|^{2}} d \eta \rightarrow 0 \quad \text { as } a \rightarrow 0^{+} .
$$

The theorem therefore follows from (6.31).

Proof of Theorem 2.5. For $b>0, \delta>0$, and $\xi \in \mathbb{R}^{n} \backslash\{0\}$ we have

$$
\begin{aligned}
\int_{\delta}^{\infty} \Phi_{\alpha, 1, b}(\xi, \tau) d \tau & =\int_{\delta}^{\infty} \frac{(\tau / b)^{\alpha-1}}{\Gamma(\alpha)} \frac{1}{(4 \pi \tau / b)^{n / 2}} e^{-\frac{b|\xi|^{2}}{4 \tau}} d \tau / b \\
& =\int_{\delta}^{\infty} \frac{1}{\Gamma(\alpha)(4 \pi)^{n / 2}}\left(\frac{\tau}{b}\right)^{\alpha-1-n / 2} e^{-\frac{b|\xi|^{2}}{4 \tau}} \frac{1}{b} d \tau \\
& =\int_{0}^{\frac{b|\xi|^{2}}{4 \delta}} \frac{1}{\Gamma(\alpha)(4 \pi)^{n / 2}}\left(\frac{|\xi|^{2}}{4 \zeta}\right)^{\alpha-1-n / 2} e^{-\zeta} \frac{|\xi|^{2}}{4 \zeta^{2}} d \zeta \\
& =\frac{\left(|\xi|^{2} / 4\right)^{\alpha-n / 2}}{\Gamma(\alpha)(4 \pi)^{n / 2}} \int_{0}^{\frac{b|\xi|^{2}}{4 \delta}} \zeta^{n / 2-\alpha-1} e^{-\zeta} d \zeta \\
& =\frac{|\xi|^{2 \alpha-n}}{4^{\alpha} \pi^{n / 2} \Gamma(\alpha)} \int_{0}^{\frac{b|\xi|^{2}}{4 \delta}} \zeta^{n / 2-\alpha-1} e^{-\zeta} d \zeta \\
& \leq\left(\frac{|\xi|^{2 \alpha-n}}{4^{\alpha} \pi^{n / 2} \Gamma(\alpha)}\right) \frac{1}{n / 2-\alpha}\left(\frac{b|\xi|^{2}}{4 \delta}\right)^{n / 2-\alpha}=C(n, \alpha)\left(\frac{b}{\delta}\right)^{n / 2-\alpha}
\end{aligned}
$$


Moreover, letting $\delta \rightarrow 0^{+}$in (6.32) we obtain

$$
\int_{0}^{\infty} \Phi_{\alpha, 1, b}(\xi, \tau) d \tau=\frac{|\xi|^{2 \alpha-n}}{\gamma(n, \alpha)} \quad \text { for } b>0 \text { and } \xi \neq 0
$$

where $\gamma$ is given in (2.14).

Let $\Omega$ be a compact subset of $\mathbb{R}^{n} \times \mathbb{R}$. Choose $R>0$ such that

$$
f=0 \quad \text { on }\left(\mathbb{R}^{n} \backslash B_{R}(0)\right) \times \mathbb{R}
$$

and

$$
\Omega \subset B_{R}(0) \times \mathbb{R} .
$$

Let $\varepsilon>0$. Since $f$ is uniformly continuous on $\mathbb{R}^{n} \times \mathbb{R}$ there exists $\delta>0$ such that

$$
|f(\eta, t-\tau)-f(\eta, t)|<\varepsilon
$$

whenever $\eta \in \mathbb{R}^{n}, t, \tau \in \mathbb{R}$, and $|\tau|<\delta$.

Let $(x, t) \in \Omega$. Then $|x|<R$ and thus for $|\xi| \geq 2 R$ we have

$$
|x-\xi| \geq|\xi|-|x|>2 R-R=R .
$$

Hence for $b>0$ we find by (6.35) and (6.34) that

$$
\begin{aligned}
& \left|\left(J_{\alpha, 1, b} f-J_{\alpha, 1,0} f\right)(x, t)\right| \\
& \leq \int_{|\xi|<2 R}\left|\int_{0}^{\infty} \Phi_{\alpha, 1, b}(\xi, \tau) f(x-\xi, t-\tau) d \tau-\frac{f(x-\xi, t)}{\gamma(n, \alpha)|\xi|^{n-2 \alpha}}\right| d \xi \\
& \left.=\int_{|\xi|<2 R} \mid \int_{0}^{\infty} \Phi_{\alpha, 1, b}(\xi, \tau)(f(x-\xi, t-\tau)-f(x-\xi, t)) d \tau\right) \mid d \xi \\
& \leq K_{1}(x, t)+K_{2}(x, t)
\end{aligned}
$$

where

$$
K_{1}(x, t)=\int_{|\xi|<2 R} \int_{0}^{\delta} \Phi_{\alpha, 1, b}(\xi, \tau)|f(x-\xi, t-\tau)-f(x-\xi, t)| d \tau d \xi
$$

and

$$
K_{2}(x, t)=\int_{|\xi|<2 R} \int_{\delta}^{\infty} \Phi_{\alpha, 1, b}(\xi, \tau)|f(x-\xi, t-\tau)-f(x-\xi, t)| d \tau d \xi .
$$

From (6.37) and (6.34) we conclude

$$
K_{1}(x, t) \leq \varepsilon \int_{|\xi|<2 R}\left(\int_{0}^{\infty} \Phi_{\alpha, 1, b}(\xi, \tau) d \tau\right) d \xi=\varepsilon \int_{|\xi|<2 R} \frac{d \xi}{\gamma(n, \alpha)|\xi|^{n-2 \alpha}}
$$

and letting $M=2\|f\|_{L^{\infty}\left(\mathbb{R}^{n} \times \mathbb{R}\right)}$ and using (6.33) we obtain

$$
\begin{aligned}
K_{2}(x, t) & \leq M \int_{|\xi|<2 R}\left(\int_{\delta}^{\infty} \Phi_{\alpha, 1, b}(\xi, \tau) d \tau\right) d \xi \\
& \leq M C(n, \alpha)\left(\frac{b}{\delta}\right)^{n / 2-\alpha}\left|B_{2 R}(0)\right| \rightarrow 0 \quad \text { as } b \rightarrow 0^{+}
\end{aligned}
$$

The theorem therefore follows from (6.38). 


\section{$7 \quad$ Preliminary results for $J_{\alpha}$ problems}

In this section we provide some lemmas needed for the proofs of our results in Section 4 dealing with solutions of the $J_{\alpha}$ problem (4.5) (4.8).

Let $\Omega=\mathbb{R}^{n} \times(a, b)$ where $n \geq 1$ and $a<b$. Lemmas 7.1 and 7.2 give estimates for the convolution

$$
\left(V_{\alpha, \Omega} f\right)(x, t)=\iint_{\Omega} \Phi_{\alpha}(x-\xi, t-\tau) f(\xi, \tau) d \xi d \tau
$$

where $\alpha>0$ and $\Phi_{\alpha}$ is defined in (2.2).

Remark 7.1. Note that if $f: \mathbb{R}^{n} \times \mathbb{R} \rightarrow \mathbb{R}$ is a nonnegative measurable function such that $\|f\|_{L^{\infty}\left(\mathbb{R}^{n} \times \mathbb{R}_{a}\right)}=0$ then

$$
V_{\alpha, \Omega} f=J_{\alpha} f \quad \text { in } \Omega:=\mathbb{R}^{n} \times(a, b) .
$$

Lemma 7.1. For $\alpha>0, \Omega=\mathbb{R}^{n} \times(a, b)$ and $f \in L^{\infty}(\Omega)$ we have

$$
\left\|V_{\alpha, \Omega} f\right\|_{L^{\infty}(\Omega)} \leq \frac{(b-a)^{\alpha}}{\Gamma(\alpha+1)}\|f\|_{L^{\infty}(\Omega)}
$$

Proof. The lemma is obvious if $\|f\|_{L^{\infty}(\Omega)}=0$. Hence we can assume $\|f\|_{L^{\infty}(\Omega)}>0$. Then for $(x, t) \in \Omega$

$$
\begin{aligned}
\frac{\left|\left(V_{\alpha, \Omega} f\right)(x, t)\right|}{\|f\|_{L^{\infty}(\Omega)}} & \leq \int_{a}^{t} \frac{(t-\tau)^{\alpha-1}}{\Gamma(\alpha)} \overbrace{\left(\int_{\xi \in \mathbb{R}^{n}} \Phi_{1}(x-\xi, t-\tau) d \xi\right)}^{=1} d \tau \\
& =-\left.\frac{(t-\tau)^{\alpha}}{\Gamma(\alpha+1)}\right|_{\tau=a} ^{\tau=t}=\frac{(t-a)^{\alpha}}{\Gamma(\alpha+1)} \\
& \leq \frac{(b-a)^{\alpha}}{\Gamma(\alpha+1)}
\end{aligned}
$$

Lemma 7.2. Let $p, q \in[1, \infty], \alpha$, and $\delta$ satisfy

$$
0 \leq \delta:=\frac{1}{p}-\frac{1}{q}<\frac{2 \alpha}{n+2}<1 .
$$

Then $V_{\alpha, \Omega}$ maps $L^{p}(\Omega)$ continuously into $L^{q}(\Omega)$ and for $f \in L^{p}(\Omega)$ we have

$$
\left\|V_{\alpha, \Omega} f\right\|_{L^{q}(\Omega)} \leq M\|f\|_{L^{p}(\Omega)}
$$

where

$$
M=C(b-a)^{\frac{2 \alpha-(n+2) \delta}{2}} \text { for some constant } C=C(n, \alpha, \delta) .
$$

Proof. Define $r \in[1, \infty)$ by

$$
1-\frac{1}{r}=\delta
$$

and define $P_{\alpha}, \bar{f}: \mathbb{R}^{n} \times \mathbb{R} \rightarrow \mathbb{R}$ by

$$
P_{\alpha}(x, t)=\Phi_{\alpha}(x, t) \chi_{(0, b-a)}(t)
$$


and

$$
\bar{f}(x, t)= \begin{cases}f(x, t) & \text { if }(x, t) \in \Omega \\ 0 & \text { elsewhere }\end{cases}
$$

Since for $t \in(a, b)$ and $\tau \in(a, t)$ we have $t-\tau \in(0, b-a)$ we see for $(x, t) \in \Omega$ that

$$
\begin{aligned}
V_{\alpha, \Omega} f(x, t) & =\int_{a}^{t} \int_{\xi \in \mathbb{R}^{n}} P_{\alpha}(x-\xi, t-\tau) f(\xi, \tau) d \xi d \tau \\
& =\iint_{\Omega} P_{\alpha}(x-\xi, t-\tau) f(\xi, \tau) d \xi d \tau \\
& =\left(P_{\alpha} * \bar{f}\right)(x, t)
\end{aligned}
$$

where $*$ is the convolution operation in $\mathbb{R}^{n} \times \mathbb{R}$.

Also since

$$
\int_{\mathbb{R}^{n}} e^{-r|x|^{2} /(4 t)} d x=\left(\frac{4 \pi t}{r}\right)^{n / 2}
$$

we have by (7.2) and (17.3) that

$$
\begin{aligned}
\left\|P_{\alpha}\right\|_{L^{r}\left(\mathbb{R}^{n} \times \mathbb{R}\right)} & =\frac{1}{\Gamma(\alpha)(4 \pi)^{n / 2}}\left(\int_{0}^{b-a} t^{r(\alpha-1-n / 2)}\left(\int_{x \in \mathbb{R}^{n}} e^{-r|x|^{2} /(4 t)} d x\right) d t\right)^{1 / r} \\
& =C(n, \alpha, r)\left(\int_{0}^{b-a} t^{r(\alpha-1-n / 2)+\frac{n}{2}} d t\right)^{1 / r} \\
& =C(n, \alpha, r)(b-a)^{\frac{2 \alpha-(n+2) \delta}{2}} .
\end{aligned}
$$

Thus by (17.4), (17.2), (17.3), and Young's inequality we have

$$
\begin{aligned}
\left\|V_{\alpha, \Omega} f\right\|_{L^{q}(\Omega)} & =\left\|P_{\alpha} * \bar{f}\right\|_{L^{q}(\Omega)} \leq\left\|P_{\alpha} * \bar{f}\right\|_{L^{q}\left(\mathbb{R}^{n} \times \mathbb{R}\right)} \\
& \leq\left\|P_{\alpha}\right\|_{L^{r}\left(\mathbb{R}^{n} \times \mathbb{R}\right)}\|\bar{f}\|_{L^{p}\left(\mathbb{R}^{n} \times \mathbb{R}\right)} \\
& \leq C(b-a)^{\frac{2 \alpha-(n+2) \delta}{2}}\|f\|_{L^{p}(\Omega)} .
\end{aligned}
$$

Lemma 7.3. Suppose $f, p$, and $K$ satisfy (4.5)-(4.8) and $(\lambda, \alpha) \in A \cup B$. Then

$$
f \in X^{\infty}
$$

Proof. Let $T>0$ be fixed. Then $f \in L^{p}\left(\mathbb{R}^{n} \times \mathbb{R}_{T}\right)$ and to complete the proof it suffices to show

$$
f \in L^{\infty}\left(\mathbb{R}^{n} \times(0, T)\right) .
$$

We consider two cases.

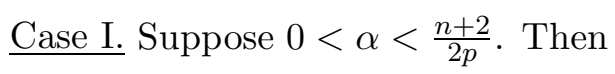

$$
0<\lambda<\frac{n+2}{n+2-2 \alpha p}
$$


and thus there exists $\varepsilon=\varepsilon(n, \lambda, \alpha, p)>0$ such that

$$
\varepsilon<2 \alpha p, \quad 2 \varepsilon<n+2-2 \alpha p, \quad \text { and } \quad \lambda<\frac{n+2}{n+2-2 \alpha p+2 \varepsilon} .
$$

Suppose

$$
f \in L^{p_{0}}\left(\mathbb{R}^{n} \times(0, T)\right) \quad \text { for some } p_{0} \in\left[p, \frac{n+2}{2 \alpha}\right) .
$$

Then letting

$$
q=\frac{(n+2) p_{0}}{n+2-2 \alpha p_{0}+\varepsilon}
$$

we have

$$
\frac{1}{p_{0}}-\frac{1}{q}=\frac{2 \alpha}{n+2}-\frac{\varepsilon}{(n+2) p_{0}} \in\left(0, \frac{2 \alpha}{n+2}\right) .
$$

Hence by (4.7), Remark 7.1, and Lemma 7.2 we see that

$$
J_{\alpha} f \in L^{q}\left(\mathbb{R}^{n} \times(0, T)\right) .
$$

Thus by (4.6) we find that

$$
0 \leq f \leq K\left(J_{\alpha} f\right)^{\lambda} \in L^{q / \lambda}\left(\mathbb{R}^{n} \times(0, T)\right)
$$

Since

$$
\begin{aligned}
\frac{q / \lambda}{p_{0}} & =\frac{n+2}{\lambda\left(n+2-2 \alpha p_{0}+\varepsilon\right)} \geq \frac{n+2-2 \alpha p+2 \varepsilon}{n+2-2 \alpha p_{0}+\varepsilon} \\
& \geq \frac{n+2-2 \alpha p+2 \varepsilon}{n+2-2 \alpha p+\varepsilon}=C(n, \lambda, \alpha, p)>1
\end{aligned}
$$

we see that starting with $p_{0}=p$ and iterating a finite number of times the process of going from (7.6) to (7.7) yields

$$
f \in L^{p_{0}}\left(\mathbb{R}^{n} \times(0, T)\right) \quad \text { for some } p_{0}>\frac{n+2}{2 \alpha} .
$$

Hence (7.5) follows from (4.6) and Lemma 7.2 .

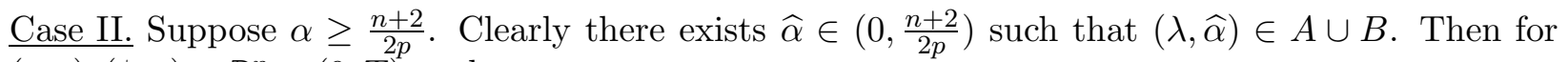
$(x, t),(\xi, \tau) \in \mathbb{R}^{n} \times(0, T)$ we have

$$
\begin{aligned}
\frac{\Phi_{\alpha}(x-\xi, t-\tau)}{\Phi_{\widehat{\alpha}}(x-\xi, t-\tau)} & =(t-\tau)^{\alpha-\widehat{\alpha}} \Gamma(\widehat{\alpha}) / \Gamma(\alpha) \\
& \leq T^{\alpha-\widehat{\alpha}} \Gamma(\widehat{\alpha}) / \Gamma(\alpha) \\
& =C(T, \alpha, \widehat{\alpha}) .
\end{aligned}
$$

Thus for $(x, t) \in \mathbb{R}^{n} \times(0, T)$ we have

$$
J_{\alpha} f(x, t) \leq C(T, \alpha, \widehat{\alpha}) J_{\widehat{\alpha}} f(x, t)
$$

and hence by (4.6) we see that

$$
0 \leq f \leq K C(T, \alpha, \widehat{\alpha})^{\lambda}\left(J_{\widehat{\alpha}} f\right)^{\lambda} \quad \text { almost everywhere in } \mathbb{R}^{n} \times(0, T) .
$$

It follows therefore from Case I that $f$ satisfies (7.5). 
Lemma 7.4. Suppose $x \in \mathbb{R}^{n}$ and $t, \tau \in(0, \infty)$ satisfy

$$
|x|^{2}<t \quad \text { and } \quad \frac{t}{4}<\tau<\frac{3 t}{4}
$$

Then

$$
\int_{|\xi|^{2}<\tau} \Phi_{1}(x-\xi, t-\tau) d \xi \geq C(n)>0
$$

where $\Phi_{\alpha}$ is defined by (2.2).

Proof. Making the change of variables $z=\frac{x-\xi}{\sqrt{4(t-\tau)}}$, letting $e_{1}=(1,0, \ldots, 0)$, and using (7.8) and (2.2) we find that

$$
\begin{aligned}
\int_{|\xi|^{2}<\tau} \Phi_{1}(x-\xi, t-\tau) d \xi & =\frac{1}{\pi^{n / 2}} \int_{\left|z-\frac{x}{\sqrt{4(t-\tau)}}\right|<\frac{\sqrt{\tau}}{\sqrt{4(t-\tau)}}} e^{-|z|^{2}} d z \\
& \geq \frac{1}{\pi^{n / 2}} \int_{\left|z-\frac{\sqrt{t}}{\sqrt{4(t-\tau)}} e_{1}\right|<\frac{\sqrt{\tau}}{\sqrt{4(t-\tau)}}} e^{-|z|^{2}} d z \\
& \geq \frac{1}{\pi^{n / 2}} \int_{\left|z-e_{1}\right|<\frac{1}{2 \sqrt{3}}} e^{-|z|^{2}} d z \\
& =C(n)>0
\end{aligned}
$$

where in this calculation we used the fact that the integral of $e^{-|z|^{2}}$ over a ball is decreased if the absolute value of the center of the ball is increased or the radius of the ball is decreased.

Lemma 7.5. For $\tau<t \leq T$ and $|x| \leq \sqrt{T-t}$ we have

$$
\int_{|\xi|<\sqrt{T-\tau}} \Phi_{1}(x-\xi, t-\tau) d \xi \geq C
$$

where $C=C(n)$ is a positive constant.

Proof. Making the change of variables $z=\frac{x-\xi}{\sqrt{t-\tau}}$ and letting $e_{1}=(1,0, \ldots, 0)$ we get

$$
\begin{aligned}
\int_{|\xi|<\sqrt{T-\tau}} \Phi_{1}(x-\xi, t-\tau) d \xi & =\frac{1}{(4 \pi)^{n / 2}} \frac{1}{(t-\tau)^{n / 2}} \int_{|\xi|<\sqrt{T-\tau}} e^{-\frac{|x-\xi|^{2}}{4(t-\tau)}} d \xi \\
& =\frac{1}{(4 \pi)^{n / 2}} \int_{\left|z-\frac{x}{\sqrt{t-\tau}}\right|<\frac{\sqrt{T-\tau}}{\sqrt{t-\tau}}} e^{-|z|^{2} / 4} d z \\
& \geq \frac{1}{(4 \pi)^{n / 2}} \int_{\left|z-\frac{\sqrt{T-\tau}}{\sqrt{t-\tau}} e_{1}\right|<\frac{\sqrt{T-\tau}}{\sqrt{t-\tau}}} e^{-|z|^{2} / 4} d z \\
& \geq \frac{1}{(4 \pi)^{n / 2}} \int_{\left|z-e_{1}\right|<1} e^{-|z|^{2} / 4} d z
\end{aligned}
$$

where the last two inequalities need some explanation. Since $|x| \leq \sqrt{T-t}<\sqrt{T-\tau}$, the center of the ball of integration in (7.9) is closer to the origin than the center of the ball of integration in (7.10). Thus, since the integrand is a decreasing function of $|z|$, we obtain (7.10). Since $\sqrt{T-\tau} \geq \sqrt{t-\tau}$, the ball of integration in (7.10) contains the ball of integration in (7.11) and hence (7.11) holds. 
Lemma 7.6. Suppose $\alpha>0, \gamma>0, p \geq 1$, and

$$
f_{0}(x, t)=\left(\frac{1}{t}\right)^{\frac{n+2}{2 p}-\gamma} \chi_{\Omega_{0}}(x, t) \quad \text { where } \Omega_{0}=\left\{(x, t) \in \mathbb{R}^{n} \times \mathbb{R}:|x|^{2}<t\right\} .
$$

Then $f_{0} \in X^{p}$ and

$$
C_{1}\left(\frac{1}{t}\right)^{\frac{n+2}{2 p}-\gamma-\alpha} \leq J_{\alpha} f_{0}(x, t) \leq C_{2}\left(\frac{1}{t}\right)^{\frac{n+2}{2 p}-\gamma-\alpha} \quad \text { for }(x, t) \in \Omega_{0}
$$

where $C_{1}$ and $C_{2}$ are positive constants depending only on $n, \alpha, \gamma$, and $p$.

Proof. For $T>0$ we have

$$
\begin{aligned}
\left\|f_{0}\right\|_{L^{p}\left(\mathbb{R}^{n} \times \mathbb{R}_{T}\right)}^{p} & =\int_{0}^{T} \int_{|x|<\sqrt{t}}\left(\frac{1}{t}\right)^{\frac{n+2}{2}-\gamma p} d x d t \\
& =C(n) \int_{0}^{T} t^{\gamma p-1} d t<\infty
\end{aligned}
$$

because $\gamma p>0$. Hence $f_{0} \in X^{p}$.

Also for $(x, t) \in \mathbb{R}^{n} \times(0, \infty)$ we have

$$
\begin{aligned}
J_{\alpha} f_{0}(x, t) & =\int_{-\infty}^{t} \int_{\xi \in \mathbb{R}^{n}} \Phi_{\alpha}(x-\xi, t-\tau) f_{0}(\xi, \tau) d \xi d \tau \\
& =\frac{1}{\Gamma(\alpha)} \int_{0}^{t}(t-\tau)^{\alpha-1}\left(\frac{1}{\tau}\right)^{\frac{n+2}{2 p}-\gamma}\left(\int_{|\xi|^{2}<\tau} \Phi_{1}(x-\xi, t-\tau) d \xi\right) d \tau
\end{aligned}
$$

Hence by Lemma 7.4 we see for $(x, t) \in \Omega_{0}$ that

$$
\begin{aligned}
J_{\alpha} f_{0}(x, t) & \geq C(n, \alpha) \int_{t / 4}^{3 t / 4}(t-\tau)^{\alpha-1}\left(\frac{1}{\tau}\right)^{\frac{n+2}{2 p}-\gamma} d \tau \\
& =C(n, \alpha) t^{\alpha-\frac{n+2}{2 p}+\gamma} \int_{1 / 4}^{3 / 4}(1-s)^{\alpha-1}\left(\frac{1}{s}\right)^{\frac{n+2}{2 p}-\gamma} d s \quad \text { where } \tau=t s \\
& =C(n, \alpha, \gamma, p) t^{\alpha-\frac{n+2}{2 p}+\gamma} .
\end{aligned}
$$

Moreover for $(x, t) \in \mathbb{R}^{n} \times(0, \infty)$ and $0<\tau<t / 2$ we have

$$
\begin{aligned}
\int_{|\xi|^{2}<\tau} \Phi_{1}(x-\xi, t-\tau) d \xi & =\frac{1}{\pi^{n / 2}} \int_{\left|z-\frac{x}{\sqrt{4(t-\tau)}}\right|<\frac{\sqrt{\tau}}{\sqrt{4(t-\tau)}}} e^{-|z|^{2}} d z \quad \text { where } z=\frac{x-\xi}{\sqrt{4(t-\tau)}} \\
& \leq \frac{\left|B_{1}(0)\right|}{\pi^{n / 2}}\left(\frac{\sqrt{\tau}}{\sqrt{4(t-\tau)}}\right)^{n}
\end{aligned}
$$

and for $(x, t) \in \mathbb{R}^{n} \times(0, \infty)$ and $t / 2<\tau<t$ we have

$$
\int_{|\xi|^{2}<\tau} \Phi_{1}(x-\xi, t-\tau) d \xi \leq \int_{\mathbb{R}^{n}} \Phi_{1}(x-\xi, t-\tau) d \xi=1 .
$$


Thus by (17.12) for $(x, t) \in \mathbb{R}^{n} \times(0, \infty)$ we have

$$
\begin{gathered}
J_{\alpha} f_{0}(x, t) \leq C(n, \alpha)\left[\int_{0}^{t / 2}(t-\tau)^{\alpha-1}\left(\frac{1}{\tau}\right)^{\frac{n+2}{2 p}-\gamma}\left(\frac{\tau}{t-\tau}\right)^{n / 2} d \tau\right. \\
\left.+\int_{t / 2}^{t}(t-\tau)^{\alpha-1}\left(\frac{1}{\tau}\right)^{\frac{n+2}{2 p}-\gamma} d \tau\right] \\
=C(n, \alpha) t^{\alpha-\frac{n+2}{2 p}+\gamma}\left[\int_{0}^{1 / 2}(1-s)^{\alpha-1}\left(\frac{1}{s}\right)^{\frac{n+2}{2 p}-\gamma}\left(\frac{s}{1-s}\right)^{n / 2} d s\right. \\
\left.\quad+\int_{1 / 2}^{1}(1-s)^{\alpha-1}\left(\frac{1}{s}\right)^{\frac{n+2}{2 p}-\gamma} d s\right] \\
=C(n, \alpha, \gamma, p) t^{\alpha-\frac{n+2}{2 p}+\gamma}
\end{gathered}
$$

because $\alpha$ and $\gamma$ are positive.

Lemma 7.7. Suppose $\alpha>0, \gamma \in \mathbb{R}, 0 \leq t_{0}<T, p \in[1, \infty)$, and

$$
f(x, t)=\left(\frac{1}{T-t}\right)^{\frac{n+2}{2 p}-\gamma} \chi_{\Omega}(x, t)
$$

where

$$
\Omega=\left\{(x, t) \in \mathbb{R}^{n} \times\left(t_{0}, T\right):|x|<\sqrt{T-t}\right\} .
$$

Then

$$
J_{\alpha} f(x, t) \geq C\left(\frac{1}{T-t}\right)^{\frac{n+2}{2 p}-\gamma-\alpha}
$$

for $(x, t) \in \Omega^{+}:=\left\{(x, t) \in \Omega: \frac{T+t_{0}}{2}<t<T\right\}$ where $C=C(n, \alpha, \gamma, p)>0$. Moreover,

$$
f \in L^{p}\left(\mathbb{R}^{n} \times \mathbb{R}\right) \text { if and only if } \gamma>0
$$

and in this case

$$
\|f\|_{L^{p}\left(\mathbb{R}^{n} \times \mathbb{R}\right)}^{p}=C(n) \int_{0}^{T-t_{0}} s^{\gamma p-1} d s
$$

Proof. Since

$$
\begin{aligned}
\|f\|_{L^{p}\left(\mathbb{R}^{n} \times \mathbb{R}\right)}^{p} & =\int_{t_{0}}^{T} \int_{|x|<\sqrt{T-t}}(T-t)^{\gamma p-\frac{n+2}{2}} d x d t \\
& =C(n) \int_{t_{0}}^{T}(T-t)^{\gamma p-1} d t=C(n) \int_{0}^{T-t_{0}} s^{\gamma p-1} d s
\end{aligned}
$$

we see that (7.13) and (17.14) hold. 
Let $r=\frac{n+2}{2 p}-\gamma-\alpha$. Then for $(x, t) \in \Omega$ we have

$$
\begin{aligned}
J_{\alpha} f(x, t) & =\int_{t_{0}}^{t}(T-\tau)^{-r-\alpha} \int_{|\xi|<\sqrt{T-\tau}} \Phi_{\alpha}(x-\xi, t-\tau) d \xi d \tau \\
& =C \int_{t_{0}}^{t}(T-\tau)^{-r-\alpha}(t-\tau)^{\alpha-1}\left(\int_{|\xi|<\sqrt{T-\tau}} \Phi_{1}(x-\xi, t-\tau) d \xi\right) d \tau \\
& \geq C \int_{t_{0}}^{t}(T-\tau)^{-r-\alpha}(t-\tau)^{\alpha-1} d \tau, \quad \text { by Lemma 7.5, } \\
& =C(T-t)^{-r} g\left(\frac{t-t_{0}}{T-t}\right)
\end{aligned}
$$

where $g(z)=\int_{0}^{z}(\zeta+1)^{-r-\alpha} \zeta^{\alpha-1} d \zeta$ and where we made the change of variables $t-\tau=(T-t) \zeta$. Thus

$$
J_{\alpha} f(x, t) \geq C(T-t)^{-r} \quad \text { for }(x, t) \in \Omega^{+}
$$

because $\frac{t-t_{0}}{T-t}>1$ in $\Omega^{+}$.

\section{Proofs of results for $J_{\alpha}$ problems}

In this section we prove our results stated in Section 4 concerning pointwise bounds for nonnegative solutions $f$ of (4.5)-(4.8). As explained in Section 4, these results immediately imply Theorems 3.13 .6 in Section 3.

Remark 8.1. The function $g: \mathbb{R}^{n} \times \mathbb{R} \rightarrow[0, \infty)$ defined by

$$
g(x, t)=g(t)= \begin{cases}\left(M t^{\alpha}\right)^{\frac{\lambda}{1-\lambda}} & \text { for } t>0 \\ 0 & \text { for } t \leq 0\end{cases}
$$

where $\alpha>0,0<\lambda<1$, and $M=M(\alpha, \lambda)$ is defined in (4.12), satisfies

$$
g=\left(J_{\alpha} g\right)^{\lambda} \text { in } \mathbb{R}^{n} \times \mathbb{R}
$$

which can be verified using (5.4). Even though $g \notin X^{p}$ for all $p \geq 1$, it will be useful in our analysis of solutions of (4.6), (4.7) which are in $X^{p}$ for some $p \geq 1$.

Remark 8.2. It will be convenient to scale (4.6) as follows. Suppose $K, \lambda, \alpha, T \in(0, \infty), \lambda \neq 1$, and $f, \bar{f}: \mathbb{R}^{n} \times \mathbb{R} \rightarrow \mathbb{R}$ are nonnegative measurable functions such that $f=\bar{f}=0$ in $\mathbb{R}^{n} \times(-\infty, 0)$ and

$$
f(x, t)=K^{\frac{1}{1-\lambda}} T^{\frac{\alpha \lambda}{1-\lambda}} \bar{f}(\bar{x}, \bar{t})
$$

where

$$
x=T^{1 / 2} \bar{x} \quad \text { and } \quad t=T \bar{t} .
$$

Then $f$ satisfies

$$
0 \leq f \leq K\left(J_{\alpha} f\right)^{\lambda} \quad \text { in } \mathbb{R}^{n} \times \mathbb{R}
$$

if and only if $\bar{f}$ satisfies

$$
0 \leq \bar{f} \leq\left(J_{\alpha} \bar{f}\right)^{\lambda} \quad \text { in } \mathbb{R}^{n} \times \mathbb{R}
$$

Moreover

$$
\frac{f(x, t)}{K^{\frac{1}{1-\lambda}} t^{\frac{\alpha \lambda}{1-\lambda}}}=\frac{\bar{f}(\bar{x}, \bar{t})}{\bar{t}^{\frac{\alpha \lambda}{1-\lambda}}} \quad \text { for }(x, t) \in \mathbb{R}^{n} \times(0, \infty)
$$


and

$$
\frac{J_{\alpha} f(x, t)}{K^{\frac{1}{1-\lambda}} t^{\frac{\alpha}{1-\lambda}}}=\frac{J_{\alpha} \bar{f}(\bar{x}, \bar{t})}{\bar{t}^{\frac{\alpha}{1-\lambda}}} \quad \text { for }(x, t) \in \mathbb{R}^{n} \times(0, \infty)
$$

Proof of Theorem 4.1. Suppose for contradiction that (4.9) is false. Then there exists $T>0$ such that

$$
\|f\|_{L^{\infty}\left(\mathbb{R}^{n} \times \mathbb{R}_{T}\right)}>0 .
$$

Hence by (4.7) there exists $t_{0} \in[0, T)$ such that

$$
\|f\|_{L^{\infty}\left(\mathbb{R}^{n} \times \mathbb{R}_{t}\right)} \begin{cases}=0 & \text { for } t \leq t_{0} \\ >0 & \text { for } t>t_{0} .\end{cases}
$$

Thus by Remark 7.1, we have for all $b>t_{0}$ that

$$
J_{\alpha} f=V_{\alpha, \Omega_{b}} f \quad \text { in } \Omega_{b}
$$

where $\Omega_{b}=\mathbb{R}^{n} \times\left(t_{0}, b\right)$ and $V_{\alpha, \Omega}$ is defined by (7.1). Also, by Lemma 7.3,

$$
\|f\|_{L^{\infty}\left(\Omega_{b}\right)} \leq\|f\|_{L^{\infty}\left(\Omega_{T}\right)}<\infty \text { for } t_{0}<b<T .
$$

It follows therefore from (4.6) and Lemma 7.1 that for $t_{0}<b<T$ we have

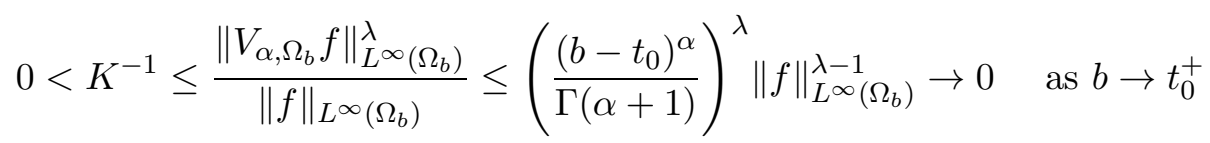

because $\lambda \geq 1$. This contradiction proves Theorem 4.1 .

Proof of Theorem 4.2. By Remark 8.2 with $T=1$ we can assume $K=1$. For $b>0$ we have by Lemma 7.3 that

$$
f \in L^{\infty}\left(\mathbb{R}^{n} \times \mathbb{R}_{b}\right)
$$

and by (4.6), (4.7), Remark 7.1 with $a=0$, and Lemma 7.1 that

$$
\|f\|_{L^{\infty}\left(\Omega_{b}\right)} \leq\left\|J_{\alpha} f\right\|_{L^{\infty}\left(\Omega_{b}\right)}^{\lambda} \leq\left(\frac{b^{\alpha}}{\Gamma(\alpha+1)}\|f\|_{L^{\infty}\left(\Omega_{b}\right)}\right)^{\lambda}
$$

where $\Omega_{b}=\mathbb{R}^{n} \times(0, b)$. Thus, since $0<\lambda<1$, we see that

$$
\|f\|_{L^{\infty}\left(\Omega_{b}\right)} \leq\left(\frac{b^{\alpha}}{\Gamma(\alpha+1)}\right)^{\frac{\lambda}{1-\lambda}} \text { for all } b>0 .
$$

Define $\left\{\gamma_{j}\right\} \subset(0, \infty)$ by $\gamma_{1}=1$ and

$$
\gamma_{j+1}=\left(\bar{M} \gamma_{j}\right)^{\lambda}, j=1,2, \ldots, \quad \text { where } \bar{M}=\Gamma(\alpha+1) M .
$$

Then, since $0<\lambda<1$, we see that

$$
\gamma_{j} \rightarrow \bar{M}^{\frac{\lambda}{1-\lambda}} \quad \text { as } j \rightarrow \infty
$$


Suppose for some positive integer $j$ that

$$
\|f\|_{L^{\infty}\left(\Omega_{b}\right)} \leq \gamma_{j}\left(\frac{b^{\alpha}}{\Gamma(\alpha+1)}\right)^{\frac{\lambda}{1-\lambda}} \text { for all } b>0 .
$$

Then for $b>0$ and $(x, t) \in \Omega_{b}$ we find from (4.6) and (5.4) that

$$
\begin{aligned}
f(x, t) & \leq\left(J_{\alpha} f(x, t)\right)^{\lambda} \\
& \leq\left(\int_{0}^{t} \frac{(t-\tau)^{\alpha-1}}{\Gamma(\alpha)}\left(\int_{\xi \in \mathbb{R}^{n}} \Phi_{1}(x-\xi, t-\tau) d \xi\right)\|f\|_{L^{\infty}\left(\Omega_{\tau}\right)} d \tau\right)^{\lambda} \\
& \leq\left(\int_{0}^{t} \frac{(t-\tau)^{\alpha-1}}{\Gamma(\alpha)} \gamma_{j}\left(\frac{\tau^{\alpha}}{\Gamma(\alpha+1)}\right)^{\frac{\lambda}{1-\lambda}} d \tau\right)^{\lambda} \\
& =\left(\gamma_{j} \frac{1}{\Gamma(\alpha) \Gamma(\alpha+1)^{\frac{\lambda}{1-\lambda}}} \int_{0}^{t}(t-\tau)^{\alpha-1} \tau^{\frac{\alpha \lambda}{1-\lambda}} d \tau\right)^{\lambda} \\
& =\left(\gamma_{j} \frac{\Gamma(\alpha) \Gamma\left(\frac{\alpha \lambda}{1-\lambda}+1\right) t^{\alpha+\frac{\alpha \lambda}{1-\lambda}}}{\Gamma(\alpha) \Gamma(\alpha+1)^{\frac{\lambda}{1-\lambda}} \Gamma\left(\alpha+\frac{\alpha \lambda}{1-\lambda}+1\right)}\right)^{\lambda} \\
& =\left(\gamma_{j} \frac{M t^{\frac{\alpha}{1-\lambda}}}{\Gamma(\alpha+1)^{\frac{\lambda}{1-\lambda}}}\right)^{\lambda}=\left(\gamma_{j} \frac{\bar{M} t^{\frac{\alpha}{1-\lambda}}}{\Gamma(\alpha+1)^{\frac{1}{1-\lambda}}}\right)^{\lambda} \\
& =\gamma_{j+1}\left(\frac{t^{\alpha}}{\Gamma(\alpha+1)}\right)^{\frac{\lambda}{1-\lambda}} \cdot
\end{aligned}
$$

Thus

$$
\|f\|_{L^{\infty}\left(\Omega_{b}\right)} \leq \gamma_{j+1}\left(\frac{b^{\alpha}}{\Gamma(\alpha+1)}\right)^{\frac{\lambda}{1-\lambda}} \text { for all } b>0 .
$$

Hence (4.10) follows inductively from (8.2)- 8.5).

Finally, repeating the calculation (8.6) with $\gamma_{j}=\gamma_{j+1}=\bar{M}^{\frac{\lambda}{1-\lambda}}$ we get

$$
\left(J_{\alpha} f(x, t)\right)^{\lambda} \leq \bar{M}^{\frac{\lambda}{1-\lambda}}\left(\frac{t^{\alpha}}{\Gamma(\alpha+1)}\right)^{\frac{\lambda}{1-\lambda}} \quad \text { for }(x, t) \in \Omega_{b}
$$

which proves (4.11).

Proof of Theorem 4.3. By Remark 8.2 we can assume $K=T=1$. For $(x, t) \in \mathbb{R}^{n} \times \mathbb{R}$ and $\delta \in(0,1)$ let

$$
g_{\delta}(x, t)=g_{\delta}(t)=\psi_{\delta}(t) g(t)
$$

where $g$ is as in Remark 8.1 and $\psi_{\delta} \in C^{\infty}(\mathbb{R} \rightarrow[0,1])$ satisfies

$$
\psi_{\delta}(t)= \begin{cases}1 & \text { if } t \leq 1 \\ 0 & \text { if } t \geq 1+\delta\end{cases}
$$


Then for $1 \leq t \leq 1+\delta$

$$
\begin{aligned}
J_{\alpha} g(t)-J_{\alpha} g_{\delta}(t) & =\int_{1}^{t} \frac{(t-\tau)^{\alpha-1}}{\Gamma(\alpha)} g(\tau)\left(1-\psi_{\delta}(\tau)\right) d \tau \\
& \leq \int_{1}^{t} \frac{(t-\tau)^{\alpha-1}}{\Gamma(\alpha)} g(\tau) d \tau \leq g(1+\delta) \int_{1}^{t} \frac{(t-\tau)^{\alpha-1}}{\Gamma(\alpha)} d \tau \\
& =g(1+\delta) \frac{(t-1)^{\alpha}}{\Gamma(\alpha+1)} \leq g(2) \frac{\delta^{\alpha}}{\Gamma(\alpha+1)}
\end{aligned}
$$

and thus by (8.1) we have for $1 \leq t \leq 1+\delta$ that

$$
\begin{aligned}
\frac{J_{\alpha} g_{\delta}(t)}{J_{\alpha} g(t)} & =\frac{J_{\alpha} g(t)-\left(J_{\alpha} g(t)-J_{\alpha} g_{\delta}(t)\right)}{g(t)^{1 / \lambda}} \\
& \geq 1-\frac{g(2) \delta^{\alpha}}{\Gamma(\alpha+1) g(1)^{1 / \lambda}} \\
& =1-C(\alpha, \lambda) \delta^{\alpha} \geq \sqrt{\frac{N}{M}}
\end{aligned}
$$

provided we choose $\delta=\delta(\alpha, \lambda, N) \in(0,1)$ sufficiently small. Hence for $1 \leq t \leq 1+\delta$ we see from (8.1) that

$$
g_{\delta}(t) \leq g(t)=\left(J_{\alpha} g(t)\right)^{\lambda} \leq\left(\frac{M}{N}\right)^{\lambda / 2}\left(J_{\alpha} g_{\delta}(t)\right)^{\lambda}
$$

which by (8.7) and (8.1) holds for all other $t$ as well.

Next let $\varphi(x)=e^{-\psi(x)}$ where $\psi(x)=\sqrt{1+|x|^{2}}-1$. Then for $\varepsilon \in(0,1), \gamma>1$, and $|\xi-x|<\gamma \sqrt{2}$ we have

$$
\frac{\varphi(\varepsilon \xi)}{\varphi(\varepsilon x)}=e^{-(\psi(\varepsilon \xi)-\psi(\varepsilon x))} \geq e^{-\varepsilon|\xi-x|} \geq e^{-\varepsilon \gamma \sqrt{2}} .
$$

Thus defining $f_{\varepsilon}: \mathbb{R}^{n} \times \mathbb{R} \rightarrow[0, \infty)$ by

$$
f_{\varepsilon}(x, t)=\varphi(\varepsilon x)\left(\frac{N}{M}\right)^{\frac{\lambda}{1-\lambda}} g_{\delta}(t)
$$

we find for $|\xi-x|<\gamma \sqrt{2}$ and $\tau \in \mathbb{R}$ that

$$
f_{\varepsilon}(\xi, \tau) \geq \varphi(\varepsilon x) e^{-\varepsilon \gamma \sqrt{2}}\left(\frac{N}{M}\right)^{\frac{\lambda}{1-\lambda}} g_{\delta}(\tau) .
$$

Thus for $(x, t) \in \mathbb{R}^{n} \times(0,2)$ we have

$$
J_{\alpha} f_{\varepsilon}(x, t) \geq \varphi(\varepsilon x) e^{-\varepsilon \gamma \sqrt{2}}\left(\frac{N}{M}\right)^{\frac{\lambda}{1-\lambda}} \int_{0}^{t} \frac{(t-\tau)^{\alpha-1}}{\Gamma(\alpha)} g_{\delta}(\tau) \int_{|\xi-x|<\gamma \sqrt{2}} \Phi_{1}(x-\xi, t-\tau) d \xi d \tau .
$$

But for $x, \xi \in \mathbb{R}^{n}$ and $0<\tau<t<2$ we find making the change of variables $z=\frac{x-\xi}{\sqrt{4(t-\tau)}}$ that

$$
\begin{aligned}
\int_{|\xi-x|<\gamma \sqrt{2}} \Phi_{1}(x-\xi, t-\tau) d \xi & \geq \int_{|\xi-x|<\gamma \sqrt{t-\tau}} \frac{1}{(4 \pi(t-\tau))^{n / 2}} e^{-\frac{|x-\xi|^{2}}{4(t-\tau)}} d \xi \\
& =\frac{1}{\pi^{n / 2}} \int_{|z|<\gamma / 2} e^{-|z|^{2}} d z=: I(\gamma) \rightarrow 1
\end{aligned}
$$


as $\gamma \rightarrow \infty$. Thus by (8.9) and (8.8) we have for $(x, t) \in \mathbb{R}^{n} \times(0,1+\delta)$ that

$$
\begin{aligned}
& \frac{\left(J_{\alpha} f_{\varepsilon}(x, t)\right)^{\lambda}}{f_{\varepsilon}(x, t)} \geq \frac{\varphi(\varepsilon x)^{\lambda} e^{-\varepsilon \gamma \lambda \sqrt{2}}\left(\frac{N}{M}\right)^{\frac{\lambda^{2}}{1-\lambda}} I(\gamma)^{\lambda}\left(J_{\alpha} g_{\delta}(t)\right)^{\lambda}}{\varphi(\varepsilon x)\left(\frac{N}{M}\right)^{\frac{\lambda}{1-\lambda}} g_{\delta}(t)} \\
& \geq\left(\frac{M}{N}\right)^{\lambda / 2} I(\gamma)^{\lambda} e^{-\varepsilon \gamma \lambda \sqrt{2}}
\end{aligned}
$$

So first choosing $\gamma$ so large that $\left(\frac{M}{N}\right)^{\lambda / 2} I(\gamma)^{\lambda}>1$ and then choosing $\varepsilon>0$ so small that (8.10) is greater than 1 we see that $f:=f_{\varepsilon}$ satisfies(4.6) in $\mathbb{R}^{n} \times(0,1+\delta)$. Thus, since $g_{\delta}(t)$ and hence $f(x, t)$ is identically zero in $\mathbb{R}^{n} \times((-\infty, 0] \cup[1+\delta, \infty))$ see that $f$ satisfies (4.6), (4.7).

From the exponential decay of $\varphi(x)$ as $|x| \rightarrow \infty$, we see that $f$ satisfies (4.13). Also since $f$ is uniformly continuous and bounded on $\mathbb{R}^{n} \times \mathbb{R}$ and

$$
\int_{a}^{b} \int_{\mathbb{R}^{n}} \Phi_{\alpha}(x, t) d x d t=\frac{1}{\Gamma(\alpha+1)}\left(b^{\alpha}-a^{\alpha}\right) \quad \text { for } a<b
$$

we easily check that (4.14) holds.

Finally, since

$$
f(0, t)=\left(\frac{N}{M}\right)^{\frac{\lambda}{1-\lambda}} g(t) \quad \text { for } 0 \leq t \leq 1
$$

we find that (4.15) holds and thus (4.16) follows from (4.6).

Proof of Theorem 4.4. By Remark 8.2 with $T=1$ we can assume $K=1$. Define $\bar{f}: \mathbb{R}^{n} \times \mathbb{R} \rightarrow[0, \infty)$ by

$$
\bar{f}(x, t)=g(t) \chi_{\left\{|x|^{2}<t\right\}}(x, t)
$$

where $g$ is defined in Remark 8.1. Then for $(x, t) \in \mathbb{R}^{n} \times(0, \infty)$ we have

$$
J_{\alpha} \bar{f}(x, t)=\int_{0}^{t} \frac{(t-\tau)^{\alpha-1}}{\Gamma(\alpha)}\left(\int_{|\xi|^{2}<\tau} \Phi_{1}(x-\xi, t-\tau) d \xi\right) g(\tau) d \tau .
$$

Thus by Lemma 7.4 we see for $|x|^{2}<t$ that

$$
\begin{aligned}
J_{\alpha} \bar{f}(x, t) & \geq C(n, \alpha, \lambda) \int_{t / 4}^{3 t / 4}(t-\tau)^{\alpha-1} \tau^{\frac{\alpha \lambda}{1-\lambda}} d \tau \\
& =C(n, \alpha, \lambda) t^{\frac{\alpha}{1-\lambda}} \\
& =C(n, \alpha, \lambda) g(x, t)^{1 / \lambda} \\
& =C(n, \alpha, \lambda) \bar{f}(x, t)^{1 / \lambda}
\end{aligned}
$$

which also holds in $\left(\mathbb{R}^{n} \times \mathbb{R}\right) \backslash\left\{|x|^{2} \leq t\right\}$ because $\bar{f}=0$ there. Thus letting $f=L \bar{f}$ where

$$
L=C^{\frac{\lambda}{1-\lambda}}
$$

where $C=C(n, \alpha, \lambda)$ is as in (8.12) we find that $f$ satisfies (4.5)-(4.7).

It follows from (8.11) and the definitions of $g$ and $f$ that there exists $N>0$ such that (4.17) holds. Thus, since $f$ solves (4.6) we obtain (4.18). 
Proof of Theorem 4.5. Since $\left|R_{j}\right|<\infty$, to prove Theorem 4.5 it suffices to show for each $\varepsilon \in(0,1)$ that the conclusion of Theorem 4.5 holds for some

$$
q \in(p, p+\varepsilon) .
$$

So let $\varepsilon \in(0,1)$. By (4.19) 1 , there exists $q$ satisfying (8.13) such that

$$
\alpha<\frac{n+2}{2 q}\left(1-\frac{1}{\lambda}\right)
$$

Define $f_{0}: \mathbb{R}^{n} \times \mathbb{R} \rightarrow \mathbb{R}$ by

$$
f_{0}(x, t)=\left(\frac{1}{t}\right)^{r} \chi_{\Omega_{0}}(x, t)
$$

where

$$
\Omega_{0}=\left\{(x, t) \in \mathbb{R}^{n} \times \mathbb{R}:|x|^{2}<t<1\right\}
$$

and

$$
r:=\frac{n+2}{2 q}<\frac{n+2}{2 p}
$$

by (8.13). Then by (8.16) and Lemma 7.6 we have

$$
f_{0} \in L^{p}\left(\mathbb{R}^{n} \times \mathbb{R}\right)
$$

and

$$
J_{\alpha} f_{0}(x, t) \geq C\left(\frac{1}{t}\right)^{r-\alpha} \quad \text { for }(x, t) \in \Omega_{0}
$$

where, throughout this entire proof, $C=C(n, \lambda, \alpha, p, q)$ is a positive constant whose value may change from line to line.

Let $\left\{T_{j}\right\} \subset(0,1 / 2)$ be a sequence such that

$$
T_{j+1}<T_{j} / 4 \quad j=1,2, \ldots
$$

and define

$$
t_{j}=T_{j} / 2
$$

Then

$$
\Omega_{j}:=\left\{(y, s) \in \mathbb{R}^{n} \times \mathbb{R}:|y|<\sqrt{T_{j}-s} \text { and } t_{j}<s<T_{j}\right\} \subset R_{j} \subset \Omega_{0}
$$

and thus defining $f_{j}: \mathbb{R}^{n} \times \mathbb{R} \rightarrow \mathbb{R}$ by

$$
f_{j}(x, t)=\left(T_{j}-t\right)^{-r} \chi_{\Omega_{j}}(x, t)
$$

we obtain from (8.16) and Lemma 7.7 that

$$
\begin{gathered}
\left\|f_{j}\right\|_{L^{p}\left(\mathbb{R}^{n} \times \mathbb{R}\right)}^{p}=C(n) \int_{0}^{T_{j}-t_{j}} s^{\left(\frac{n+2}{2 p}-r\right) p-1} d s \rightarrow 0 \quad \text { as } j \rightarrow \infty, \\
\left\|f_{j}\right\|_{L^{q}\left(R_{j}\right)}=\left\|f_{j}\right\|_{L^{q}\left(\mathbb{R}^{n} \times \mathbb{R}\right)}=\infty \quad \text { for } j=1,2, \ldots,
\end{gathered}
$$

and

$$
J_{\alpha} f_{j}(x, t) \geq C\left(\frac{1}{\left(T_{j}-t\right)}\right)^{r-\alpha} \quad \text { for }(x, t) \in \Omega_{j}^{+}
$$


where

$$
\Omega_{j}^{+}=\left\{(x, t) \in \Omega_{j}: \frac{3 T_{j}}{4}<t<T_{j}\right\} .
$$

It follows from (8.15) and (8.18) that

$$
\frac{f_{0}(x, t)}{\left(J_{\alpha} f_{0}(x, t)\right)^{\lambda}} \leq C t^{(r-\alpha) \lambda-r} \quad \text { for }(x, t) \in \Omega_{0}
$$

and from (8.14) and (8.16) that the exponent

$$
(r-\alpha) \lambda-r=\lambda[r(1-1 / \lambda)-\alpha]>0 .
$$

Thus

$$
\sup _{\Omega_{0}} \frac{f_{0}}{\left(J_{\alpha} f_{0}\right)^{\lambda}} \leq C
$$

and by (8.20)

$$
\sup _{\Omega_{j}} \frac{f_{0}}{\left(J_{\alpha} f_{0}\right)^{\lambda}} \leq C T_{j}^{(r-\alpha) \lambda-r}<1
$$

by taking a subsequence.

By (8.21), (8.24), and (8.25) we have

$$
\begin{aligned}
\sup _{\Omega_{j}^{+}} \frac{f_{j}}{\left(J_{\alpha} f_{j}\right)^{\lambda}} & \leq C \sup _{(x, t) \in \Omega_{j}^{+}}\left(T_{j}-t\right)^{(r-\alpha) \lambda-r} \\
& \leq C\left(T_{j}-t_{j}\right)^{(r-\alpha) \lambda-r}<1
\end{aligned}
$$

by taking a subsequence.

It follows from (8.15), (8.21), (8.20), and (8.19) that

$$
\sup _{\Omega_{j}} \frac{f_{0}}{f_{j}}=\sup _{(x, t) \in \Omega_{j}} \frac{\left(T_{j}-t\right)^{r}}{t^{r}} \leq \frac{\left(T_{j}-t_{j}\right)^{r}}{t_{j}^{r}}=1
$$

and letting $\Omega_{j}^{-}=\Omega_{j} \backslash \Omega_{j}^{+}$we see from (8.21), (8.18), (8.20), and (8.25) that

$$
\begin{aligned}
\sup _{\Omega_{j}^{-}} \frac{f_{j}}{\left(J_{\alpha} f_{0}\right)^{\lambda}} & \leq C \sup _{(x, t) \in \Omega_{j}^{-}} \frac{t^{(r-\alpha) \lambda}}{\left(T_{j}-t\right)^{r}} \leq C \frac{T_{j}^{(r-\alpha) \lambda}}{\left(T_{j} / 4\right)^{r}} \\
& =C T_{j}^{(r-\alpha) \lambda-r}<\frac{1}{2}
\end{aligned}
$$

by taking a subsequence.

Taking an appropriate subsequence of $f_{j}$ and letting

$$
f=f_{0}+\sum_{j=1}^{\infty} f_{j}
$$

we find from (8.17) and (8.22) that $f$ satsfies (4.20).

In $\Omega_{j}^{+}$we have by (8.27) and (8.28) that

$$
\begin{aligned}
f & =f_{0}+f_{j} \leq\left(J_{\alpha} f_{0}\right)^{\lambda}+\left(J_{\alpha} f_{j}\right)^{\lambda} \\
& \leq\left(J_{\alpha}\left(f_{0}+f_{j}\right)\right)^{\lambda} \leq\left(J_{\alpha} f\right)^{\lambda} .
\end{aligned}
$$


In $\Omega_{j}^{-}$we have by (8.29) and (8.30) that

$$
f=f_{0}+f_{j} \leq 2 f_{j} \leq\left(J_{\alpha} f_{0}\right)^{\lambda} \leq\left(J_{\alpha} f\right)^{\lambda} .
$$

In $\Omega_{0} \backslash \cup_{j=1}^{\infty} \Omega_{j}$ we have by (8.26) that

$$
f=f_{0} \leq C\left(J_{\alpha} f_{0}\right)^{\lambda} \leq C\left(J_{\alpha} f\right)^{\lambda} .
$$

In $\left(\mathbb{R}^{n} \times \mathbb{R}\right) \backslash \Omega_{0}, f=0 \leq\left(J_{\alpha} f\right)^{\lambda}$. Thus, after scaling $f$, we see that $f$ is a solution of (4.6), (4.7). Also (4.21) holds by (8.23).

Proof of Theorem 4.6. By (4.23) 1 , there exists a unique number $\gamma \in\left(0, \frac{n+2}{2 p}-\alpha\right)$ such that

$$
\lambda=\frac{\frac{n+2}{2 p}-\gamma}{\frac{n+2}{2 p}-\alpha-\gamma} .
$$

Let $f_{0}$ and $\Omega_{0}$ be as in Lemma 7.6. Then by (8.31) and Lemma 7.6 we have

$$
f_{0} \in X^{p}
$$

and

$$
f_{0} \leq C\left(J_{\alpha} f_{0}\right)^{\lambda} \quad \text { in } \mathbb{R}^{n} \times \mathbb{R}
$$

where in this proof $C=C(n, \lambda, \alpha, p)$ is a positive constant whose value may change from line to line. Let $\left\{T_{j}\right\},\left\{t_{j}\right\} \subset(2, \infty)$ satisfy

$$
T_{j+1} \geq 4 T_{j} \quad \text { and } \quad T_{j}=2 t_{j}
$$

and define $f_{j}: \mathbb{R}^{n} \times \mathbb{R} \rightarrow \mathbb{R}$ by

$$
f_{j}(x, t)=\left(\frac{1}{T_{j}-t}\right)^{\frac{n+2}{2 p}-\gamma} \chi_{\Omega_{j}}(x, t)
$$

where

$$
\Omega_{j}:=\left\{(x, t) \in \mathbb{R}^{n} \times\left(T_{j} / 2, T_{j}\right):|x|<\sqrt{T_{j}-t}\right\} .
$$

Then

$$
\begin{gathered}
\Omega_{j} \subset R_{j} \subset \Omega_{0}, \quad \Omega_{j} \cap \Omega_{k}=\emptyset \quad \text { for } j \neq k, \\
\inf \left\{t:(x, t) \in \Omega_{j}\right\}=T_{j} / 2 \rightarrow \infty \quad \text { as } j \rightarrow \infty,
\end{gathered}
$$

and by (8.34), (8.31), and Lemma 7.7 we have

$$
f_{j} \in L^{p}\left(\mathbb{R}^{n} \times \mathbb{R}\right)
$$

and

$$
f_{j} \leq C\left(J_{\alpha} f_{j}\right)^{\lambda} \quad \text { in } \Omega_{j}^{+}
$$

where

$$
\Omega_{j}^{+}=\left\{(x, t) \in \Omega_{j}: \frac{3 T_{j}}{4}<t<T_{j}\right\} .
$$

It follows therefore from (8.33) that

$$
f_{0}+f_{j} \leq C\left(\left(J_{\alpha} f_{0}\right)^{\lambda}+\left(J_{\alpha} f_{j}\right)^{\lambda}\right) \leq C\left(J_{\alpha}\left(f_{0}+f_{j}\right)\right)^{\lambda} \quad \text { in } \Omega_{j}^{+} .
$$


In $\Omega_{j}^{-}:=\Omega_{j} \backslash \Omega_{j}^{+}$we have

$$
\frac{f_{j}}{f_{0}}=\left(\frac{t}{\left(T_{j}-t\right)}\right)^{\frac{n+2}{2 p}-\gamma} \leq\left(\frac{\frac{3}{4} T_{j}}{\frac{1}{4} T_{j}}\right)^{\frac{n+2}{2 p}-\gamma}=3^{\frac{n+2}{2 p}-\gamma}
$$

and thus we obtain from (8.33) that

$$
f_{0}+f_{j} \leq C f_{0} \leq C\left(J_{\alpha} f_{0}\right)^{\lambda} \leq C\left(J_{0}\left(f_{0}+f_{j}\right)\right)^{\lambda} \quad \text { in } \Omega_{j}^{-} .
$$

Let $f=f_{0}+\sum_{j=1}^{\infty} f_{j}$. Then clearly $f$ satisfies (4.7) and by (8.32), (8.37), and (8.36) we see that $f$ satisfies (4.24).

In $\Omega_{j}$ we have by (8.35) 2 , (8.38), and (8.39) that

$$
f=f_{0}+f_{j} \leq C\left(J_{\alpha}\left(f_{0}+f_{j}\right)\right)^{\lambda} \leq C\left(J_{\alpha} f\right)^{\lambda}
$$

and in $\left(\mathbb{R}^{n} \times \mathbb{R}\right) \backslash \cup_{j=1}^{\infty} \Omega_{j}$ we have by (8.33) that

$$
f=f_{0} \leq C\left(J_{\alpha} f_{0}\right)^{\lambda} \leq C\left(J_{\alpha} f\right)^{\lambda} .
$$

Thus after scaling $f$, we find that $f$ satisfies (4.6).

Since $\left|R_{j}\right|<\infty$, we can for the proof of (4.25) assume instead of (4.23) 2 that

$$
q=\frac{n+2}{2 \alpha}\left(1-\frac{1}{\lambda}\right)
$$

and hence by (8.31) we get

$$
\frac{n+2}{2 p}-\gamma=\frac{\alpha}{1-\frac{1}{\lambda}}=\frac{n+2}{2 q} .
$$

Consequently from (8.35) 1 , (8.34), and Lemma 7.7 we find that

$$
\|f\|_{L^{q}\left(R_{j}\right)} \geq\left\|f_{j}\right\|_{L^{q}\left(\Omega_{j}\right)}=\infty \quad \text { for } j=1,2, \ldots
$$

which proves (4.25)

\section{A Appendix}

For the proof of Theorem 2.3(ii) we will need the following result due to Nogin and Rubin [19] concerning the inversion of the operator $J_{\alpha}$ in the framework of the spaces $L^{p}\left(\mathbb{R}^{n} \times \mathbb{R}\right)$. See also [24, Theorem 9.24].

Theorem A.1. Suppose $0<\alpha<\frac{n+2}{2 p}, 1<p<\infty$, and $u=J_{\alpha} f$ with $f \in L^{p}\left(\mathbb{R}^{n} \times \mathbb{R}\right)$. Then

$$
\lim _{\varepsilon \rightarrow 0^{+}} J_{\varepsilon}^{-\alpha} u=f \quad \text { in } L^{p}\left(\mathbb{R}^{n} \times \mathbb{R}\right)
$$

where

$$
J_{\varepsilon}^{-\alpha} u(x, t)=C(n, \alpha, l) \iint_{\mathbb{R}^{n} \times(\varepsilon, \infty)} \frac{\left(\Delta_{y, \tau}^{l} u\right)(x, t)}{\tau^{1+\alpha}} e^{-\frac{|y|^{2}}{4}} d y d \tau
$$

and

$$
\left(\Delta_{y, \tau}^{l} u\right)(x, t)=\sum_{k=0}^{l}(-1)^{k}\left(\begin{array}{l}
l \\
k
\end{array}\right) u(x-y \sqrt{k \tau}, t-k \tau), \quad l>\alpha .
$$

\section{Acknowledgments}

The author thanks the anonymous referee for very helpful comments. 


\section{References}

[1] B. Abdellaoui, A. Attar, R. Bentifour, I. Peral, On fractional p-Laplacian parabolic problem with general data, Ann. Mat. Pura Appl. (4) 197 (2018) 329-356.

[2] E. Affili, E. Valdinoci, Decay estimates for evolution equations with classical and fractional time-derivatives, J. Differential Equations, https://doi.org/10.1016/j.jde.2018.09.031

[3] Boumediene Abdellaoui, Maria Medina, Ireneo Peral, Ana Primo, Optimal results for the fractional heat equation involving the Hardy potential, Nonlinear Anal. 140 (2016) 166-207.

[4] Mark Allen, A nondivergence parabolic problem with a fractional time derivative, Differential Integral Equations 31 (2018) 215-230.

[5] Mark Allen, Luis Caffarelli, Alexis Vasseur, A parabolic problem with a fractional time derivative, Arch. Ration. Mech. Anal. 221 (2016) 603-630.

[6] Ioannis Athanasopoulos, Luis Caffarelli, Emmanouil Milakis, On the regularity of the nondynamic parabolic fractional obstacle problem, J. Differential Equations 265 (2018) 2614-2647.

[7] Matteo Bonforte, Juan Luis Vázquez, A priori estimates for fractional nonlinear degenerate diffusion equations on bounded domains, Arch. Ration. Mech. Anal. 218 (2015) 317-362.

[8] Huyuan Chen, Laurent Véron, Ying Wang, Fractional heat equations with subcritical absorption having a measure as initial data, Nonlinear Anal. 137 (2016) 306-337.

[9] Matías G. Delgadino, Scott Smith, Hölder estimates for fractional parabolic equations with critical divergence free drifts, Ann. Inst. H. Poincar Anal. Non Linaire 35 (2018) 577-604.

[10] S. Dipierro, E. Valdinoci, V. Vespri, Decay estimates for evolutionary equations with fractional time-diffusion, J. Evol. Equ. https://doi.org/10.1007/s00028-019-00482-z

[11] Giulia Furioli, Tatsuki Kawakami, Bernhard Ruf, Elide Terraneo, Asymptotic behavior and decay estimates of the solutions for a nonlinear parabolic equation with exponential nonlinearity, J. Differential Equations 262 (2017) 145-180.

[12] Ciprian G. Gal, Mahamadi Warma, On some degenerate non-local parabolic equation associated with the fractional p-Laplacian, Dyn. Partial Differ. Equ. 14 (2017) 47-77.

[13] V. R. Gopala Rao, A characterization of parabolic function spaces, Amer. J. Math. 99 (1977) 985-993.

[14] Mohamed Jleli, Bessem Samet, The decay of mass for a nonlinear fractional reaction-diffusion equation, Math. Methods Appl. Sci. 38 (2015) 1369-1378.

[15] Jan Kadlec, Solution of the first boundary value problem for a generalization of the heat equation in classes of functions possessing a fractional derivative with respect to the timevariable, (Russian) Czechoslovak Math. J. 16 (91) (1966) 91-113.

[16] Jukka Kemppainen, Juhana Siljander, Vicente Vergara, Rico Zacher, Decay estimates for timefractional and other non-local in time subdiffusion equations in $\mathbb{R}^{d}$. Math. Ann. 366 (2016) 941-979 
[17] M. Mirzazadeh, Analytical study of solitons to nonlinear time fractional parabolic equations. Nonlinear Dynam. 85 (2016) 2569-2576.

[18] Luc Molinet, Slim Tayachi, Remarks on the Cauchy problem for the one-dimensional quadratic (fractional) heat equation, J. Funct. Anal. 269 (2015) 2305-2327.

[19] V. A. Nogin, B. S. Rubin, The spaces $L_{p, r}^{\alpha}\left(R^{n+1}\right)$ of parabolic potentials, (Russian) Anal. Math. 13 (1987) 321-338.

[20] K. Nyström, O. Sande, Extension properties and boundary estimates for a fractional heat operator, Nonlinear Anal. 140 (2016) 29-37.

[21] Ebru Ozbilge, Ali Demir, Identification of unknown coefficient in time fractional parabolic equation with mixed boundary conditions via semigroup approach, Dynam. Systems Appl. 24 (2015) 341-348.

[22] Fabio Punzo, Enrico Valdinoci, Uniqueness in weighted Lebesgue spaces for a class of fractional parabolic and elliptic equations, J. Differential Equations 258 (2015) 555-587.

[23] Walter Rudin, Functional analysis, McGraw-Hill Series in Higher Mathematics, McGraw-Hill Book Co., New York-Düsseldorf-Johannesburg, 1973.

[24] Stefan G. Samko, Hypersingular integrals and their applications, Analytical Methods and Special Functions, 5, Taylor \& Francis, Ltd., London, 2002.

[25] Charles H. Sampson, A Characterization of Parabolic Lebesgue Spaces, Dissertation, Rice Univ., 1968.

[26] Elias M. Stein, Singular integrals and differentiability properties of functions, Princeton Mathematical Series, No. 30, Princeton University Press, Princeton, N.J. 1970.

[27] Pablo Raúl Stinga, José L. Torrea, Regularity theory and extension problem for fractional nonlocal parabolic equations and the master equation, SIAM J. Math. Anal. 49 (2017) 38933924 .

[28] Fuqin Sun, Peihu Shi, Global existence and non-existence for a higher-order parabolic equation with time-fractional term, Nonlinear Anal. 75 (2012) 4145-4155.

[29] Vladimir Varlamov, Long-time asymptotics for the nonlinear heat equation with a fractional Laplacian in a ball, Studia Math. 142 (2000) 71-99.

[30] Juan Luis Vázquez, Bruno Volzone, Symmetrization for linear and nonlinear fractional parabolic equations of porous medium type, J. Math. Pures Appl. (9) 101 (2014) 553-582.

[31] Juan Luis Vázquez, Arturo de Pablo, Fernando Quirós, Ana Rodríguez, Classical solutions and higher regularity for nonlinear fractional diffusion equations, J. Eur. Math. Soc. 19 (2017) 1949-1975.

[32] Vicente Vergara, Rico Zacher, Optimal decay estimates for time-fractional and other nonlocal subdiffusion equations via energy methods, SIAM J. Math. Anal. 47 (2015) 210-239.

[33] Quan-Guo Zhang, Hong-Rui Sun, The blow-up and global existence of solutions of Cauchy problems for a time fractional diffusion equation, Topol. Methods Nonlinear Anal. 46 (2015) 69-92. 